Radius of curvature estimation and localization of targets using multiple sonar sensors

Billur Barshan, and Ali şafak Sekmen

Citation: The Journal of the Acoustical Society of America 105, 2318 (1999); doi: 10.1121/1.426838

View online: http://dx.doi.org/10.1121/1.426838

View Table of Contents: http://asa.scitation.org/toc/jas/105/4

Published by the Acoustical Society of America 


\title{
Radius of curvature estimation and localization of targets using multiple sonar sensors
}

\author{
Billur Barshan \\ Department of Electrical Engineering, Bilkent University, Bilkent, 06533 Ankara, Turkey
}

\author{
Ali Şafak Sekmen \\ Center for Intelligent Systems, Department of Electrical and Computer Engineering, Vanderbilt University, \\ Box 1824, Station B, Nashville, Tennessee 37235
}

(Received 12 August 1998; accepted 31 December 1998)

\begin{abstract}
Acoustic sensors have been widely used in time-of-flight ranging systems since they are inexpensive and convenient to use. One of the most important limitations of these sensors is their low angular resolution. To improve the angular resolution and the accuracy, a novel, flexible, and adaptive three-dimensional (3-D) multi-sensor sonar system is described for estimating the radius of curvature and location of cylindrical and spherical targets. Point, line, and planar targets are included as limiting cases which are important for the characterization of typical environments. Sensitivity analysis of the curvature estimate with respect to measurement errors and certain system parameters is provided. The analysis and the simulations are verified by experiments in 2-D with specularly reflecting cylindrical and planar targets, using a real sonar system. Typical accuracies in range and azimuth are $0.18 \mathrm{~mm}$ and $0.1^{\circ}$, respectively. Accuracy of the curvature estimation depends on the target type and system parameters such as transducer separation and operating range. The adaptive configuration brings an improvement varying between $35 \%$ and $45 \%$ in the accuracy of the curvature estimate. The presented results are useful for target differentiation and tracking applications. (C) 1999 Acoustical Society of America. [S0001-4966(99)03904-1]

PACS numbers: 43.58.-e, 43.28.Tc, 43.60.Qv, 43.35.Yb [SLE]
\end{abstract}

\section{INTRODUCTION}

Ultrasonic transducers are a convenient and inexpensive means for intelligent systems to build models of their environment. However, these sensors are limited by their wide beamwidth which makes accurate localization of targets difficult. To increase the localization accuracy, an adaptive sensor configuration composed of multiple ultrasonic transducers is proposed that is capable of estimating the radius of curvature and location of spheres, cylinders, point, line, and planar targets. Consequently, these basic types of reflectors can be differentiated.

Target localization has been extensively studied in earlier work. In Ref. 1, time-delay estimation for active/passive localization in underwater sonar is reviewed with references to benchmark work. In particular, ocean effects which require sonar adaptation are considered. Adaptive sonar arrays have been also used by other researchers to add flexibility to their systems. ${ }^{2}$ Coherent and incoherent processing techniques of time-delay estimation have been addressed in Refs. 3, 4. Active, wide-band detection and localization of targets in a dense and uncertain multipath environment has been considered in Ref. 5. The review article in Ref. 6 considers numerical schemes for accurate processing of information from both active and passive acoustic arrays.

Sonar sensing has many applications for intelligent systems operating in three-dimensional (3-D) environments, such as airborne or underwater robots. Several researchers have investigated the limitations of sonar for 3-D target recognition, discrimination, and tracking: Self-contained navigation systems have been devised for underwater vehicles, capable of tracking and producing continuous range information from a passive target. ${ }^{7}$ In Ref. 8 , an approach is described to the construction of 3-D stochastic models for intelligent systems exploring the underwater environment. In Ref. 9, the minimum amount of information and actuation needed to track a ball in 3-D has been determined and implemented using qualitative methods. Hong and Kleeman have investigated the geometry of 3-D corner cubes using a lowsample rate equilateral triangular sonar configuration. ${ }^{10}$ Kleeman and Akbarally have classified and discriminated the target primitives commonly occurring in 3-D space. ${ }^{11}$ Peremans et al. ${ }^{12}$ and Sabatini ${ }^{13,14}$ both have investigated curved reflectors using linear array configurations. In Ref. 15, an analytical approach to surface curvature extraction is described which employs ultrasonic echo trajectories and differential geometry. In Refs. 16 and 17, binaural sonar information is fused for accurate object recognition using a system which adaptively changes its position and configuration in response to the echoes it detects. Curvature estimation has been also important in image analysis to provide viewpoint-independent cues for shape classification. ${ }^{18}$

Some sonar systems attempt to emulate the remarkable perception and pattern recognition capabilities of bats and dolphins in extracting detailed information about their environments from acoustic echo returns. ${ }^{19-21}$ Artificial neural networks have been widely used for this purpose, to process time and/or frequency representations of sonar echo signals. For example, one application is in the classification of sonar returns from undersea targets where the targets may be made of different materials, have different shape, buried in mud or sediment, or exist in the presence of other reflectors in the 
environment. $^{21-23}$ In Ref. 24, cylinder-wall thicknesses discriminating capability of artificial neural networks is compared to that of dolphins. In Ref. 25, artificial neural networks are applied to classifying underwater active sonar returns with different numbers of peaks. Another system can recognize 3-D cubes and tetrahedrons, independent of their orientation with the help of neural networks. ${ }^{19}$

Acoustic imaging of extended targets by means of synthetic-aperture sonar has been considered in Ref. 26, where echoes from spherical and cylindrical targets laid down on a seabed are processed together with random echoes from the sea bottom. In Ref. 27, Stergiopoulos reviews the implementation of adaptive synthetic-aperture processing schemes in integrated active-passive sonar systems.

In this paper, an adaptive sonar configuration is used for radius of curvature estimation and localization of targets. When the reflection point of the target is not along the lineof-sight of the transducer, the amplitude of the reflected signal is smaller, which decreases the signal-to-noise ratio (SNR) and worsens the accuracy. To reduce this effect, the transducers are rotated toward the target to obtain more nearly accurate estimates.

The organization of the paper is as follows: In Sec. I, background information on acoustic reflection and signal models of sonar sensors is reviewed and motivation for the adaptive configuration is provided. Methods for time-offlight estimation are discussed in Sec. II. In Sec. III, the geometry of reflection from spherical targets is considered and analyzed for radius of curvature estimation. The important limiting cases of point and planar targets are highlighted. Sensitivity analysis of curvature estimation is provided with respect to measurement errors and variations in some of the system parameters in Sec. IV. Section V presents the simulation results. A detailed description of the sensing device used in this study is provided in Sec. VIA. Experimental results which verify the analysis and the simulations are presented in Sec. VIB. Section VII briefly discusses the use of the method for target differentiation. Finally, conclusions are drawn and directions for future work are motivated.

\section{ACOUSTIC REFLECTION AND SIGNAL MODELS}

The characteristics of the radiation pattern of an acoustic transducer are different in the near-field (or Fresnel) region and the far-field (or Fraunhofer) region. ${ }^{28}$ In this study, assuming all targets of interest are located in the far field, the far-field model of a piston-type transducer having a circular aperture is used. ${ }^{28}$ For a single frequency of excitation, the far-field characteristics at range $z$ and angular deviation $\alpha$ from the line-of-sight are described by ${ }^{29,30}$

$$
p_{z, \alpha}=\frac{p_{\max } z_{\min }}{z} \frac{J_{1}(k a \sin \alpha)}{k a \sin \alpha} \text { for } z \geqslant z_{\min },
$$

where $J_{1}($.$) is the Bessel function of the first order of the$ first kind and $p_{\max }$ is the propagation pressure amplitude on the beam axis at range $z_{\min }$ along the line-of-sight. $z_{\min }$ $\cong a^{2} / \lambda$ is the distance at which the far-zone characteristics begin. Although the 2-D cross-section of the characteristics is given here, in fact, the pattern is rotationally symmetric about the line-of-sight.

The half beamwidth angle $\alpha_{0}$ in the far-field corresponds to the first zero of the Bessel function in Eq. (1) which occurs at $k a \sin \alpha_{0}=1.22 \pi$, resulting in: ${ }^{31}$

$$
\alpha_{0}=\sin ^{-1}\left[\frac{0.61 \lambda}{a}\right]
$$

where $\lambda=c / f_{0}$ is the wavelength ( $f_{0}$ is the resonance frequency of the transducer) and $a$ is the transducer aperture radius.

Since a range of frequencies around $f_{0}$ are transmitted, the corresponding beam patterns are superposed and the resulting pattern can be approximated by a Gaussian function centered at zero with standard deviation $\sigma_{\alpha}=\alpha_{0} / 2:^{32}$

$$
\tilde{p}_{z, \alpha}=\frac{p_{\max } z_{\min }}{z} e^{-\alpha^{2} / 2 \sigma_{\alpha}^{2}} \text { for } z \geqslant z_{\min } .
$$

For a rigid cylindrical target of infinite height at range $z$ and making an angle $\alpha$ with the line-of-sight of the transducer, the received time signal can be modeled by: ${ }^{33}$

$$
\begin{aligned}
s_{z, \alpha}(t)= & \rho_{c} \frac{A_{\max } z_{\min }^{3 / 2}}{z^{3 / 2}} e^{-\alpha^{2} / \sigma_{\alpha}^{2}} e^{-\left[t-\left(t_{0}+\Delta t_{c}\right)\right]^{2} / 2 \sigma_{t}^{2}} \\
& \times \sin \left[2 \pi f_{0}\left(t-t_{0}\right)\right] \quad \text { for } z \geqslant z_{\min },
\end{aligned}
$$

where $\rho_{c}$ is the reflection coefficient which increases with the radius of curvature, ${ }^{34} A_{\text {max }}$ is the maximum signal amplitude, $z$ is the distance between the transducer and the object surface, $t_{0}$ is the time-of-flight, $\Delta t_{c}$ is the time difference between the center of the Gaussian window and $t_{0}$, and $\sigma_{t}$ $=1 / f_{0}$. Basically, the received signal envelope has been modeled as a Gaussian function centered at $t_{0}+\Delta t_{c}$ with suitably chosen variance $\sigma_{t}^{2} \cdot{ }^{33}$ More generally, the model

$$
\begin{aligned}
s_{z, \alpha}(t)= & k(z) e^{-\alpha^{2} / \sigma_{\alpha}^{2}} e^{-\left[t-\left(t_{0}+\Delta t_{c}\right)\right]^{2} / 2 \sigma_{t}^{2}} \\
& \times \sin \left[2 \pi f_{0}\left(t-t_{0}\right)\right] \text { for } z \geqslant z_{\min }
\end{aligned}
$$

is capable of representing observed signals for a wide variety of target types and locations in the far zone. ${ }^{33}$ Here, $k(z)$ incorporates $A_{\max }$ and $\rho_{c}$, and is inversely proportional to some power of the range $z$ depending on target type. ${ }^{35}$ The inclination angle $\alpha$ from the line-of-sight is related to the target azimuth and elevation angles $\theta$ and $\phi$ by the relation $\alpha=\cos ^{-1}(\cos \theta \cos \phi)$.

With a single stationary transducer, it is not possible to estimate the angular position of the target $(\theta, \phi)$ with better resolution than the angular resolution of the transducer which is approximately $2 \alpha_{0}$. When a range reading is received, all that is known is that the object lies somewhere on a spherical cap subtending a cone of half angle $\alpha_{0}$ and radius $z$, centered at the transducer. This is illustrated in Fig. 1 in 2-D for the transducer in the middle. To improve the angular resolution, the present system employs multiple identical acoustic transducers with center-to-center separation $d$ (Fig. 1). Each transducer can operate both as transmitter and as receiver and detect echo signals reflected from targets within its sensitivity region. All members of the sensor configuration can detect targets located within the joint sensitivity re- 


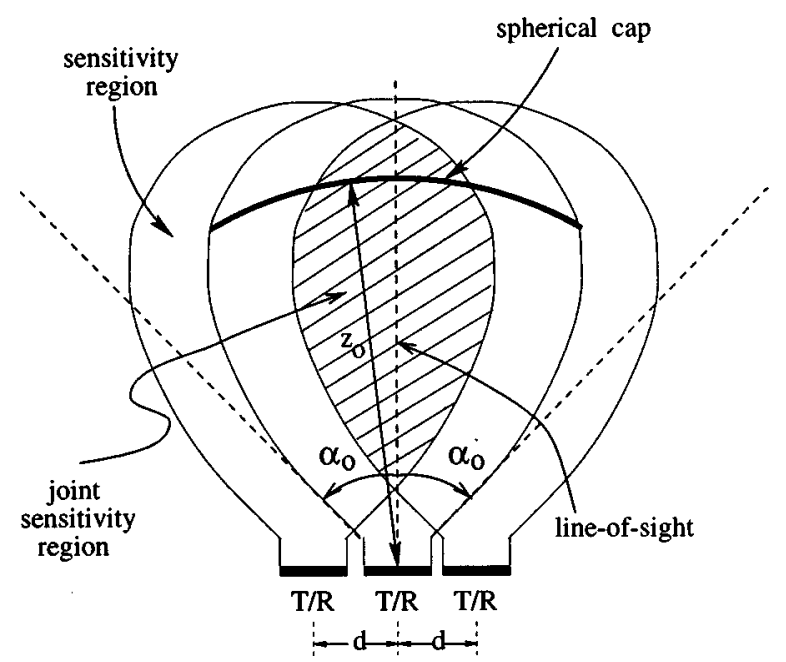

FIG. 1. Minimum distance at which a target is detectable by all three transducers is approximately $\left[(d-a) / \tan \alpha_{0}\right]+\left(a^{2} / \lambda\right)$. This corresponds to the distance between the central transducer and the start of the joint sensitivity region.

gion, which is the overlap of the individual sensitivity regions, as shown in Fig. 1. The extent of this region is different for different targets which, in general, exhibit different reflection properties. For example, for edgelike or polelike targets, this region is much smaller but of similar shape, and for planes, it is more extended. ${ }^{36}$

When the object is not located along the line-of-sight of the transducer (i.e., $\alpha \neq 0^{\circ}$ ), there is an exponential decline in the amplitude that decreases the SNR [Eq. (4)]. Hence, information provided by sonar sensors is most reliable when the object lies along the line-of-sight of the transducer. Likewise, at nearby ranges, due to the $k(z)$ term inversely varying with $z$ in Eq. (5), more nearly accurate readings are provided. Therefore, the transducers are rotated around their centers to align the line-of-sight with the object as shown in Fig. 2 in 2-D.

\section{TIME-OF-FLIGHT ESTIMATION}

The most commonly used sonar ranging systems are based on time-of-flight measurements. In thresholding timeof-flight systems, an echo is produced when a transmitted pulse encounters an object and a range reading $z=c t_{0} / 2$ is produced when the echo amplitude first exceeds a preset

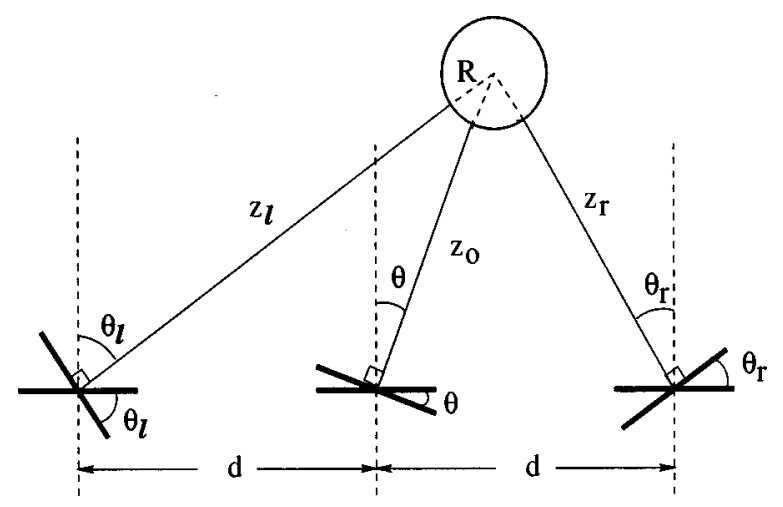

FIG. 2. The spherical object and the initial (flat) and adapted (rotated) sensor configurations illustrated in 2-D.

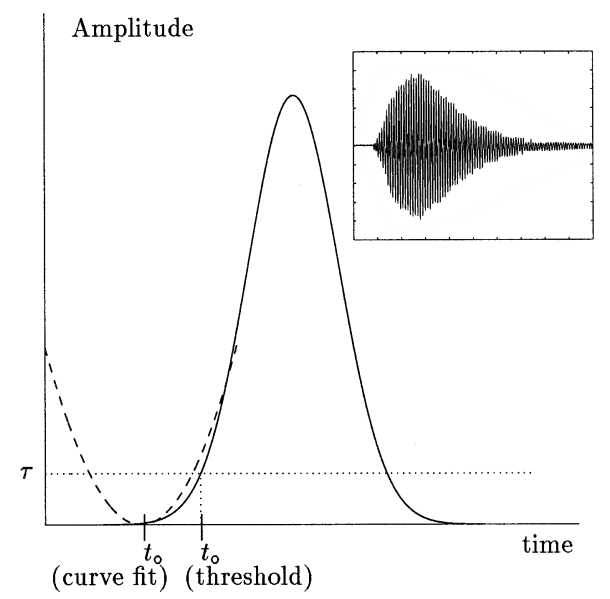

FIG. 3. Envelope of the sonar echo and time-of-flight estimation by thresholding and curve-fitting. Inset: Typical real sonar waveform.

threshold level $\tau$ back at the receiver. Here, $t_{0}$ is the timeof-flight estimate of the echo signal and $c$ is the speed of sound in air. ( $c=331.4 \sqrt{T / 273} \mathrm{~m} / \mathrm{s}$, where $T$ is the absolute temperature in Kelvin. At room temperature, $c=343.3 \mathrm{~m} / \mathrm{s}$.)

An alternative to simple thresholding is the curve-fitting method which is based on fitting a parabola to the onset of the sonar echo in order to reduce the bias on the time-offlight estimate ${ }^{35,36}$ (Fig. 3). In this method, the estimate of $t_{0}$, initially obtained by simple thresholding, is further improved by using Levenberg-Marguardt nonlinear least-squares optimization. $^{37}$

Recently, a consolidated comparison of simple thresholding, curve-fitting several variations of the sliding-window used in radar, and cross correlation methods has been provided in terms of their bias, variance, and processing time. ${ }^{38}$ The first three methods are much simpler and faster, while offering a variety of attractive compromises between accuracy and system complexity. For instance, curve-fitting improves the bias of the time-of-flight estimate but the variance is comparable and the implementation requires more effort compared to thresholding.

When operated in the pulse-echo mode, ${ }^{39}$ the transducers offer complete flexibility in the firing sequence and timing at which the individual transducers can be fired. In the firing pattern used here, each transducer registers only the echo of the signal transmitted by itself. Assuming the target is stationary, the firing is done sequentially to avoid crosstalk between the transducers. This is geometrically simpler to analyze as compared to firing patterns where the transducers simultaneously detect signals transmitted by each other. After each transmission, the detected waveform is recorded and thresholded to obtain a round-trip time-of-flight estimate.

\section{TARGET REFLECTION GEOMETRY}

In the following analysis, a stationary spherical target of radius $R$ is assumed to be present at spherical coordinates $(r, \theta, \phi)$. 


\section{A. Spherical target with radius $R$}

According to the firing pattern described in the previous section, the geometry of Fig. 2 indicates that the noisy distance measurements at the surrounding transducers are:

$$
\begin{aligned}
& \hat{z}_{0}=\frac{c \hat{t}_{0}}{2}=r-R+n_{0}, \\
& \hat{z}_{r}=\frac{c \hat{t}_{r}}{2}=\sqrt{r^{2}+d^{2}-2 d r \cos \phi \sin \theta}-R+n_{r}, \\
& \hat{z}_{l}=\frac{c \hat{t}_{l}}{2}=\sqrt{r^{2}+d^{2}+2 d r \cos \phi \sin \theta}-R+n_{l}, \\
& \hat{z}_{u}=\frac{c \hat{t}_{u}}{2}=\sqrt{r^{2}+d^{2}-2 d r \sin \phi}-R+n_{u}, \\
& \hat{z}_{d}=\frac{c \hat{t}_{d}}{2}=\sqrt{r^{2}+d^{2}+2 d r \sin \phi}-R+n_{d},
\end{aligned}
$$

where $\hat{t}_{0}, \hat{t}_{r}, \hat{t}_{l}, \hat{t}_{u}, \hat{t}_{d}$ are the time-of-flight measurements at the middle, right, left, up, and down transducers, respectively, $n_{0}, n_{r}, n_{l}, n_{u}, n_{d}$ are spatially and temporally uncorrelated zero-mean Gaussian noise. Each measurement confines the possible target locations to a spherical cap defined by the intersection of the sensitivity region and a sphere centered at the corresponding transducer (Fig. 1). At least three measurements are necessary to identify the curvature of the target both in 2-D and 3-D. This is illustrated in Figs. 4 and 5.

In Ref. 12, it has been shown that for the Polaroid transducer, the noise correlation coefficient is negligible since most of the noise on the range measurements is dominated by the thermal noise in the electronics. This is the reason why $n_{0}, n_{r}, n_{l}, n_{u}$, and $n_{d}$ can be modeled as uncorrelated Gaussian noise. Therefore, the error correlation matrix $\mathbf{C}$ and its inverse are approximated as diagonal:

$$
\mathbf{C}=\left[\begin{array}{ccccc}
\sigma_{n_{0}}^{2} & 0 & 0 & 0 & 0 \\
0 & \sigma_{n_{r}}^{2} & 0 & 0 & 0 \\
0 & 0 & \sigma_{n_{l}}^{2} & 0 & 0 \\
0 & 0 & 0 & \sigma_{n_{u}}^{2} & 0 \\
0 & 0 & 0 & 0 & \sigma_{n_{d}}^{2}
\end{array}\right],
$$

and the conditional probability density function of the measurement vector $\hat{\mathbf{m}}$ is:

$$
\begin{aligned}
p(\hat{\mathbf{m}} \mid r, \theta, \phi, R) & \\
= & \frac{1}{2 \pi|\mathbf{C}|} \exp \left\{-\frac{1}{2}[\hat{\mathbf{m}}-\mathbf{z}(r, \theta, \phi, R)]^{\mathbf{T}} \mathbf{C}^{-1}\right. \\
& \times[\hat{\mathbf{m}}-\mathbf{z}(r, \theta, \phi, R)]\},
\end{aligned}
$$

where the vectors $\hat{\mathbf{m}}$ and $\mathbf{z}(r, \theta, R)$ are defined as:

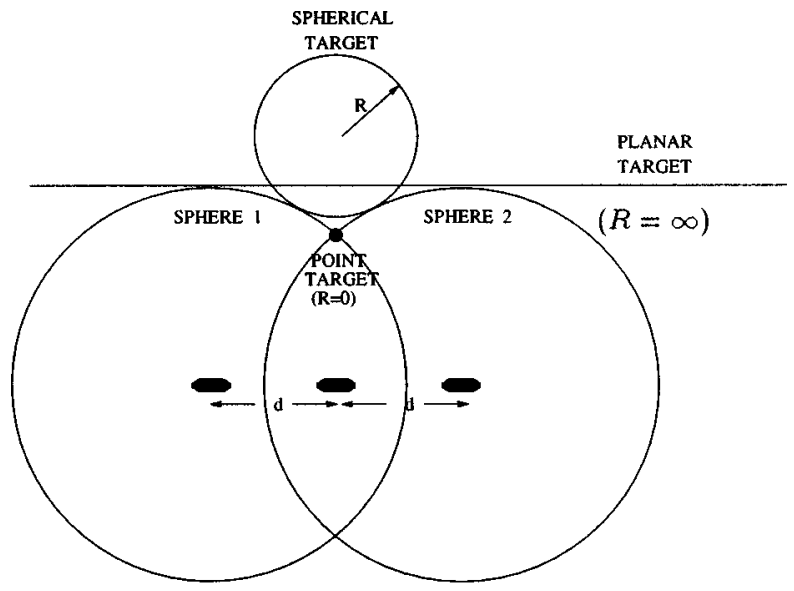

FIG. 4. The indeterminacy of curvature with only two measurements. The unknown target can have any curvature from zero to infinity.

$$
\begin{gathered}
\hat{\mathbf{m}} \triangleq\left[\begin{array}{c}
\hat{z}_{0} \\
\hat{z}_{r} \\
\hat{z}_{l} \\
\hat{z}_{u} \\
\hat{z}_{d}
\end{array}\right], \\
\mathbf{z}(r, \theta, \phi, R) \triangleq\left[\begin{array}{c}
r-R \\
\sqrt{r^{2}+d^{2}-2 d r \cos \phi \sin \theta}-R \\
\sqrt{r^{2}+d^{2}+2 d r \cos \phi \sin \theta}-R \\
\sqrt{r^{2}+d^{2}-2 d r \sin \phi}-R \\
\sqrt{r^{2}+d^{2}+2 d r \sin \phi}-R
\end{array}\right] .
\end{gathered}
$$

The $r, \theta, \phi$, and $R$ values maximizing Eq. (8) are the maximum likelihood estimates which can be found by solving $\hat{\mathbf{m}}=\mathbf{z}(\hat{r}, \hat{\theta}, \hat{\phi}, \hat{R})$ for $\hat{R}, \hat{r}, \hat{\theta}$, and $\hat{\phi}$ :

$$
\begin{aligned}
& \hat{R}=\frac{\left(\hat{z}_{r}^{2}+\hat{z}_{l}^{2}\right)-2\left(\hat{z}_{0}^{2}+d^{2}\right)}{4 \hat{z}_{0}-2\left(\hat{z}_{r}+\hat{z}_{l}\right)}=\frac{\left(\hat{z}_{u}^{2}+\hat{z}_{d}^{2}\right)-2\left(\hat{z}_{0}^{2}+d^{2}\right)}{4 \hat{z}_{0}-2\left(\hat{z}_{u}+\hat{z}_{d}\right)}, \\
& \hat{r}=\hat{z}_{0}+\hat{R}, \\
& \hat{\theta}=\sin ^{-1}\left[\frac{\left(\hat{z}_{l}-\hat{z}_{r}\right)\left(\hat{z}_{l}+\hat{z}_{r}+2 \hat{R}\right)}{\sqrt{16 d^{2}\left(\hat{z}_{0}+\hat{R}\right)^{2}-\left(\hat{z}_{d}-\hat{z}_{u}\right)^{2}\left(\hat{z}_{d}+\hat{z}_{u}+2 \hat{R}\right)^{2}}}\right], \\
& \hat{\phi}=\sin ^{-1}\left[\frac{\left(\hat{z}_{d}-\hat{z}_{u}\right)\left(\hat{z}_{d}+\hat{z}_{u}+2 \hat{R}\right)}{4 d\left(\hat{z}_{0}+\hat{R}\right)}\right] .
\end{aligned}
$$

To localize the center of a target with finite radius, one needs three measurements in 2-D and five in 3-D as evident from the above expressions for the polar coordinates of the target.

\section{B. Point target: The limit $\boldsymbol{R} \rightarrow \mathbf{0}$}

In the limit $R \rightarrow 0$, a point target is obtained. Point-target localization in 2-D has been considered in Ref. 40 and two methods of estimating the location have been presented using a linear array of transducers. The equations in 3-D derived above for finite $R$ become simpler in the limit $R \rightarrow 0$ : 


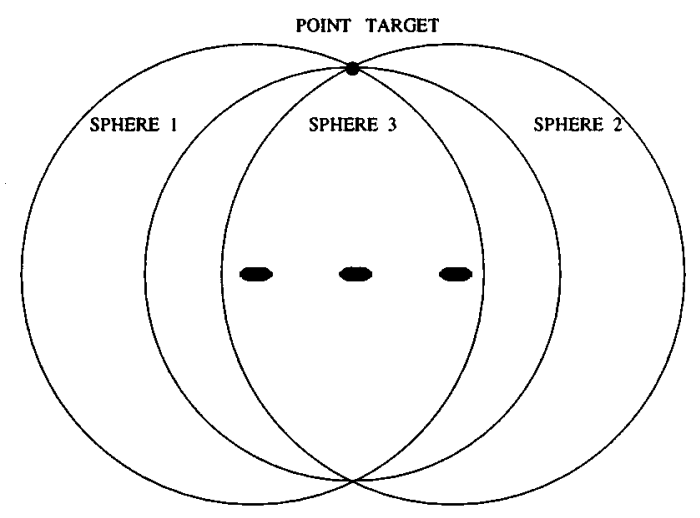

Characterizing the point-target response of a sensor is important not only for its application to point or edgelike targets, but also to assess its performance on extended targets. There are different approaches for modeling extended targets. ${ }^{12,41,42}$ If the approach is one of hypothesis testing or one of parameterizing the extended target, then sensor performance may not be easily related to its point-target response. On the other hand, for extended targets of unknown shape with possible roughness, ${ }^{32}$ point-target analysis can be extremely useful.

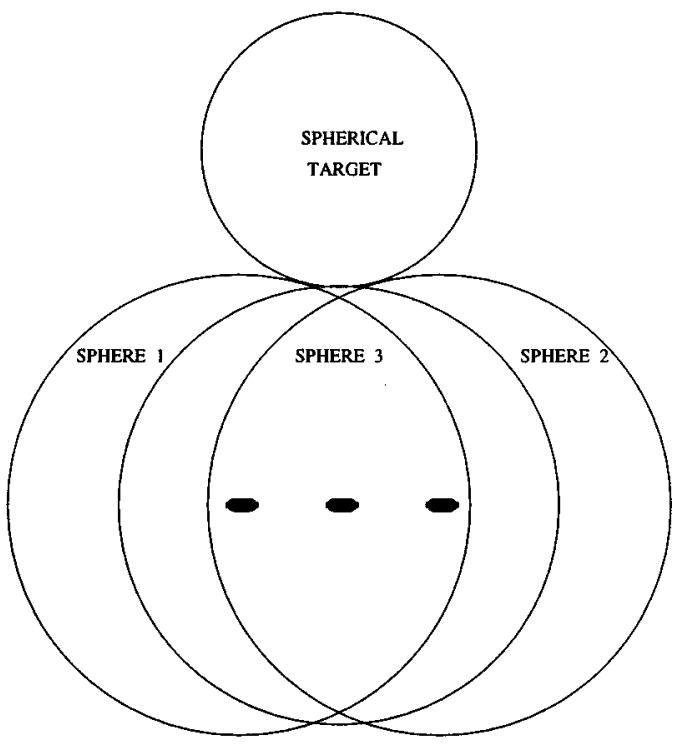

\section{Planar target: The limit $\boldsymbol{R} \rightarrow \infty$}

In the limit $R \rightarrow \infty$, the target becomes a plane. Both the distance to the center of the "sphere" and its radius of curvature become infinity. In this case, either the limits of the above equations can be taken, or more simply, the perpendicular distances of the transducers to the plane can be directly derived from the geometry. With the measurement noise taken into account:

$$
\begin{aligned}
& \hat{z}_{0}=z_{0}+n_{0}, \\
& \hat{z}_{r}=z_{0}-d \cos \phi \sin \theta+n_{r}, \\
& \hat{z}_{l}=z_{0}+d \cos \phi \sin \theta+n_{l}, \\
& \hat{z}_{u}=z_{0}-d \sin \phi+n_{u}, \\
& \hat{z}_{d}=z_{0}+d \sin \phi+n_{d} .
\end{aligned}
$$

The solution is:

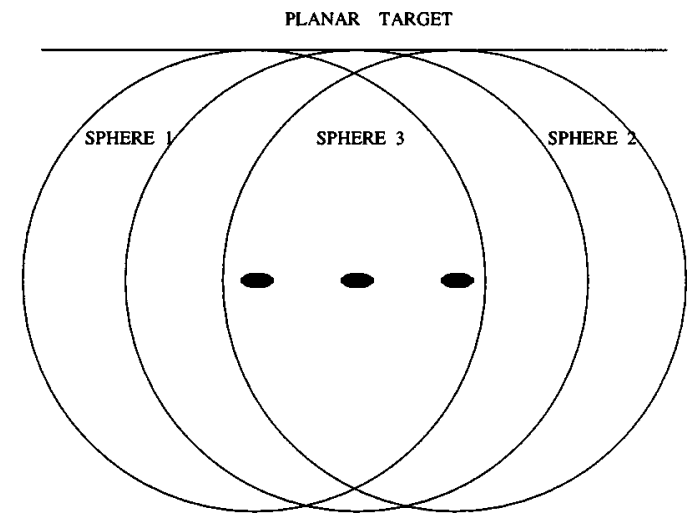

$$
\begin{aligned}
& \hat{R}=\frac{\left(\hat{z}_{r}^{2}+\hat{z}_{l}^{2}\right)-2\left(\hat{z}_{0}^{2}+d^{2}\right)}{4 n_{0}-2\left(n_{r}+n_{l}\right)} \cong \infty, \\
& \hat{r}=\hat{z}_{0}+\hat{R} \cong \infty, \\
& \hat{\theta}=\sin ^{-1}\left[\frac{\hat{z}_{l}-\hat{z}_{r}}{\sqrt{4 d^{2}-\left(\hat{z}_{d}-\hat{z}_{u}\right)^{2}}}\right], \\
& \hat{\phi}=\sin ^{-1}\left[\frac{\hat{z}_{d}-\hat{z}_{u}}{2 d}\right] .
\end{aligned}
$$

FIG. 5. Measurement geometry for point, spherical and planar targets. Three measurements uniquely identify the curvature of the unknown target both in 2-D and 3-D.

$$
\begin{aligned}
& \hat{r}=\hat{z}_{0}=\sqrt{\frac{\hat{z}_{r}^{2}+\hat{z}_{l}^{2}}{2}-d^{2}}=\sqrt{\frac{\hat{z}_{u}^{2}+\hat{z}_{d}^{2}}{2}-d^{2},} \\
& \hat{\theta}=\sin ^{-1}\left[\frac{\left(\hat{z}_{l}^{2}-\hat{z}_{r}^{2}\right)}{\sqrt{16 d^{2} \hat{z}_{0}^{2}-\left(\hat{z}_{d}^{2}-\hat{z}_{u}^{2}\right)^{2}}}\right] \\
& \hat{\phi}=\sin ^{-1}\left[\frac{\left(\hat{z}_{d}^{2}-\hat{z}_{u}^{2}\right)}{4 d \hat{z}_{0}}\right] .
\end{aligned}
$$

\section{SENSITIVITY ANALYSIS OF RADIUS OF CURVATURE}

A sensitivity analysis has been performed to determine how much variation would result in the radius of curvature estimate $R$ as a result of measurement errors and variation in certain system parameters such as $z_{0}, z_{l}, z_{r}, d, R$, and $\theta$. Since $R$ can be estimated using either the set of measurements $z_{0}, z_{r}, z_{l}$ or the set of measurements $z_{0}, z_{u}, z_{d}$, and since the equations involved are identical in form [Eq. (10)], the sensitivity of $R$ to $z_{r}\left(z_{l}\right)$ is the same as its sensitivity to $z_{u}\left(z_{d}\right)$. Furthermore, since the curvature estimation equation is symmetric with respect to $z_{r}$ and $z_{l}$ (and the alternative 
equation is symmetric with respect to $z_{u}$ and $z_{d}$ ), the sensitivity of the curvature with respect to the individual measurements $z_{r}, z_{l}, z_{u}, z_{d}$ will be identical. Therefore, it is sufficient to carry out the sensitivity analysis for only one of these distance measurements, which is chosen to be $z_{r}$.

Given the radius of curvature estimate in Eq. (10), perturbation is added to the variable for which sensitivity analysis is made. For example, the perturbation $\Delta z_{0}$ is added to $z_{0}$ and its effect on the radius of curvature estimate is calculated as follows:

$$
\begin{aligned}
\Delta R= & R\left(z_{0}+\Delta z_{0}, z_{r}, z_{l}, d\right)-R\left(z_{0}, z_{r}, z_{l}, d\right) \\
= & \frac{\left(z_{r}^{2}+z_{l}^{2}\right)-2\left[\left(z_{0}+\Delta z_{0}\right)^{2}+d^{2}\right]}{4\left(z_{0}+\Delta z_{0}\right)-2\left(z_{r}+z_{l}\right)} \\
& -\frac{\left(z_{r}^{2}+z_{l}^{2}\right)-2\left(z_{0}^{2}+d^{2}\right)}{4 z_{0}-2\left(z_{r}+z_{l}\right)} .
\end{aligned}
$$

In Fig. 6(a), Eq. (17) has been plotted for $r$ between 0 and $1.5 \mathrm{~m}$. The perturbation error $\Delta z_{0}$ on $z_{0}$ has been varied between 0 and $0.4 \mathrm{~mm}$. Actually, the typical standard deviation of the range measurement error in the current system is approximately $0.18 \mathrm{~mm}$ as verified in Sec. VI B. A stationary spherical target with radius $7.5 \mathrm{~cm}$ is assumed to be present at $\theta=0^{\circ}, \phi=0^{\circ}$. Transducer separation is set to its minimum value of $7.5 \mathrm{~cm}$ corresponding to the worst resolution case. For fixed transducer separation, error in radius of curvature increases linearly with $z_{0}$ but nonlinearly with increasing $r$. At a target range of $r=50 \mathrm{~cm}$, an error of $\Delta z_{0}$ $=0.18 \mathrm{~mm}$ on $z_{0}$ corresponds to an error of $\Delta R=15.4 \mathrm{~mm}$ on $R$, which represents about $20 \%$ error. At $r=1.0 \mathrm{~m}$, the same perturbation error on $z_{0}$ corresponds to an error of $\Delta R=61.0 \mathrm{~mm}$ on curvature, representing $82 \%$ error.

The fact that a positive error $\Delta z_{0}$ leads to a positive error $\Delta R$ can be explained as follows: Assuming that the target has convex curvature, increasing $z_{0}$ while keeping $z_{r}$ and $z_{l}$ constant corresponds to an increase in the radius of curvature $R$, or a decrease in curvature $1 / R$. For fixed $\Delta z_{0}$, error in $R$ also increases with range $r=z_{0}+R$, since the fixed transducer separation $d$ provides poorer resolution as the range increases. For larger ranges, as the ratio $d / r$ decreases, the system has smaller resolution for the given separation.

In Fig. 6(b), the sensitivity of $R$ with respect to $z_{r}$ (or equivalently $z_{l}, z_{u}, z_{d}$ ) has been plotted for the same parameters used in generating Fig. 6(a). Note that, in this case, for a positive error $\Delta z_{r}$ or $\Delta z_{l}$, the error $\Delta R$ on radius of curvature is negative. This again can be explained by the geometry of Fig. 2. A positive error on the right and left measurements, with $z_{0}$ constant, causes a reduction in the radius of curvature.

Fig. 7(a) illustrates the effect of transducer separation $d$ on the accuracy of the radius of curvature estimate. For $r$ between 0 and $1.5 \mathrm{~m}$ and $\Delta z_{0}=0.18 \mathrm{~mm}$, Eq. (17) has been plotted for transducer separations between 4.0 and $60 \mathrm{~cm}$. Corresponding plot for $\Delta z_{r}=0.18 \mathrm{~mm}$ is presented in Fig. 8(a). In both figures, it is observed that the ratio $d / r$ is a significant parameter in the curvature estimation process.

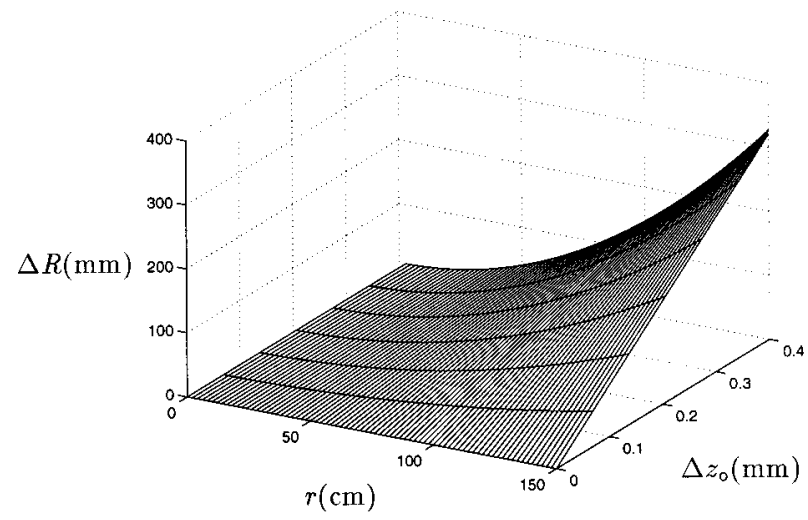

(a)

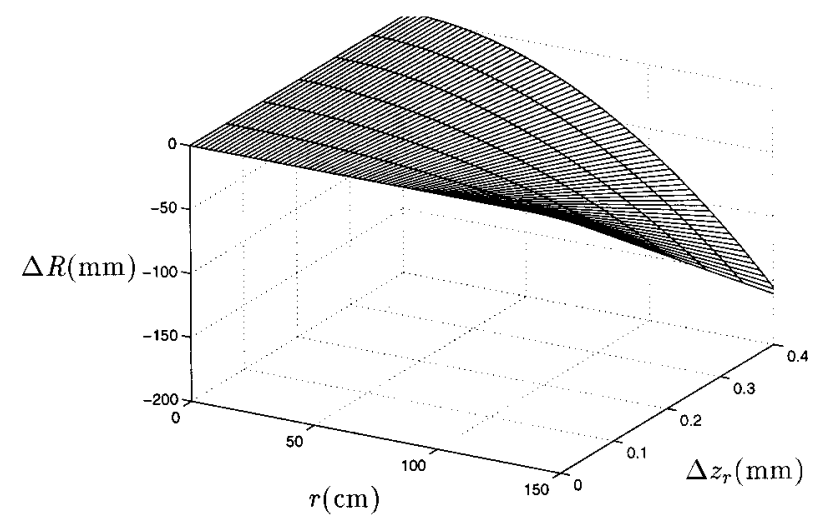

(b)

FIG. 6. Sensitivity of $R$ to distance measurements (a) $z_{0}$; (b) $z_{r}$ or $z_{l}$.

This ratio cannot be selected too large. This is because the directional sensitivity regions of the transducers are limited to a cone with half angle $\alpha_{0}$ (in the current system, $\alpha_{0}$ $\cong 12^{\circ}$, Ref. 39). If $d / r$ is selected too large, the sensitivity regions of the transducers will not overlap at the location of the target so that targets nearer than $\left[(d-a) / \tan \alpha_{0}\right]$ $+\left(a^{2} / \lambda\right)$ will not be detected. ${ }^{43}$ This approximately corresponds to the distance between the central transducer and the start of the joint sensitivity region in Fig. 1. On the other hand, if $d / r$ is selected too small, the resolution provided by the differential time-of-flight information between the central and surrounding transducers will not be sufficiently large to estimate the curvature reliably. Hence, as the operating range increases, a larger transducer separation must be used in order to maintain the same accuracy in curvature estimation. This is verified by the experimental results in Sec. VIB where it can be observed that if the range is increased while the transducer separation is kept constant, the error on $R$ increases. Thus, it is concluded that a sensor system which is to operate over a large range of target distances must have the capability of adaptively adjusting transducer separation $d$. The information provided by Figs. 1 and 6(a) can be combined to formulate a rule for choosing the optimal transducer separation $d$ for a given range $r$. Thus, one can envisage a two-step curvature estimation process: The range estimate obtained in the first step is used to adjust the transducer 


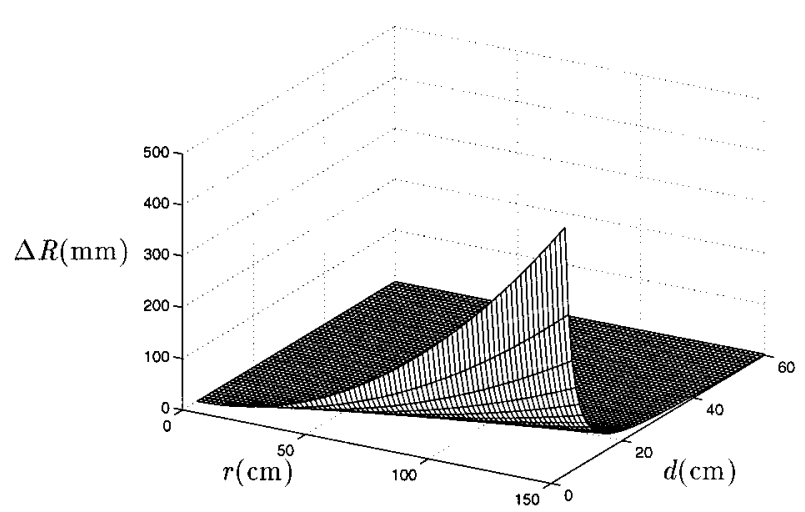

(a)

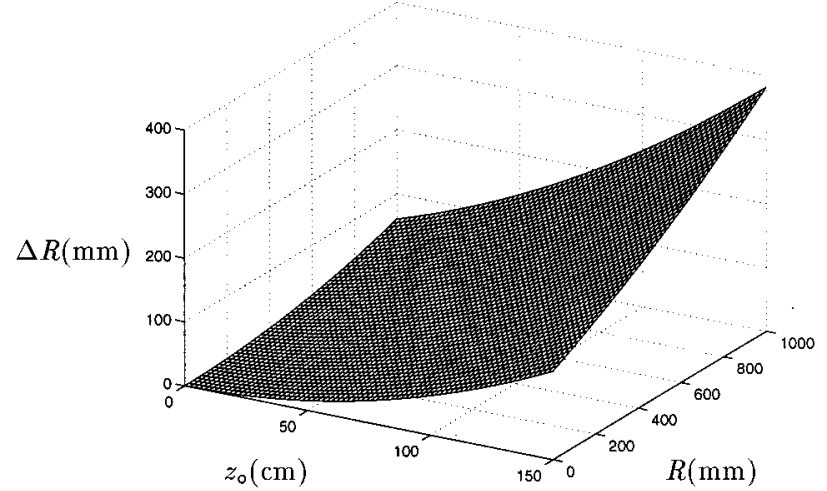

(b)

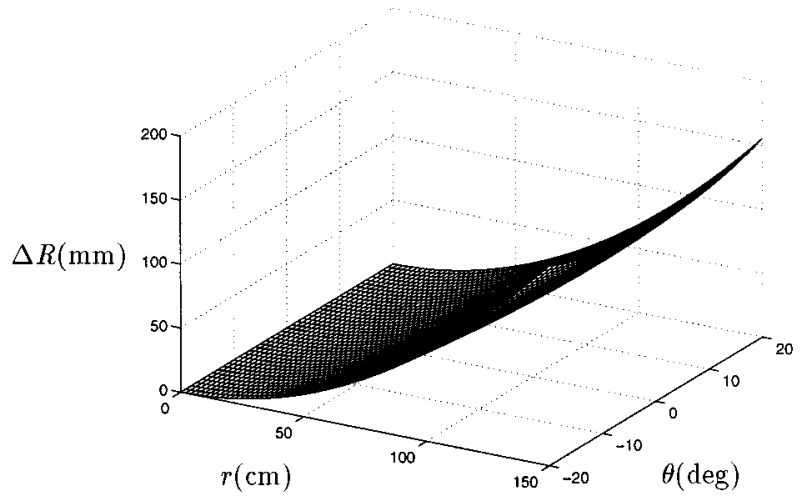

(c)

FIG. 7. Sensitivity of $R$ to (a) $d$, (b) $R$ and (c) $\theta$ when $\Delta z_{0}=0.18 \mathrm{~mm}$.

separation $d$ to its optimal value, allowing an accurate curvature estimate in the second step.

The sensitivity of curvature to a measurement error $\Delta z_{0}$ at different curvature and azimuth values has also been investigated when $\Delta z_{0}=0.18 \mathrm{~mm}$. In the first case, it is observed that for fixed $z_{0}, \Delta R$ increases with the radius of curvature of the target as illustrated in Fig. 7(b). In Fig. 7(c), $\theta$ is varied from $-20^{\circ}$ to $20^{\circ}$ with $d=7.5 \mathrm{~cm}$. It can be observed that there is a slight increase in $\Delta R$ with increasing $|\theta|$. Corresponding plots for a perturbation $\Delta z_{r}=0.18 \mathrm{~mm}$ on the radius of curvature estimate are presented in Fig. 8(b) and (c).

As will also be confirmed experimentally in Sec. VIB, accuracy is much less of a problem with the localization parameters so that a sensitivity analysis is not presented for these parameters.

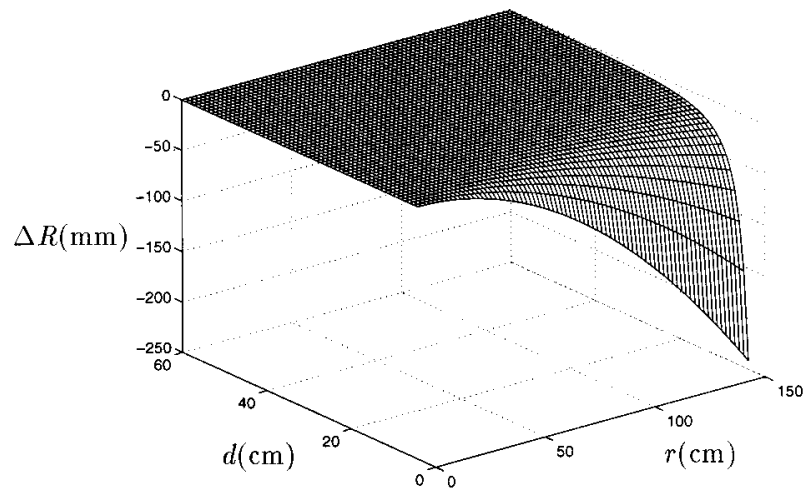

(a)

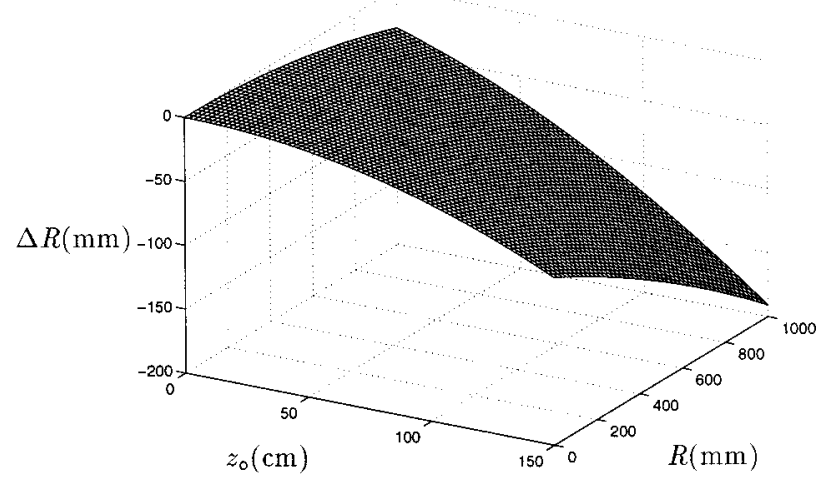

(b)

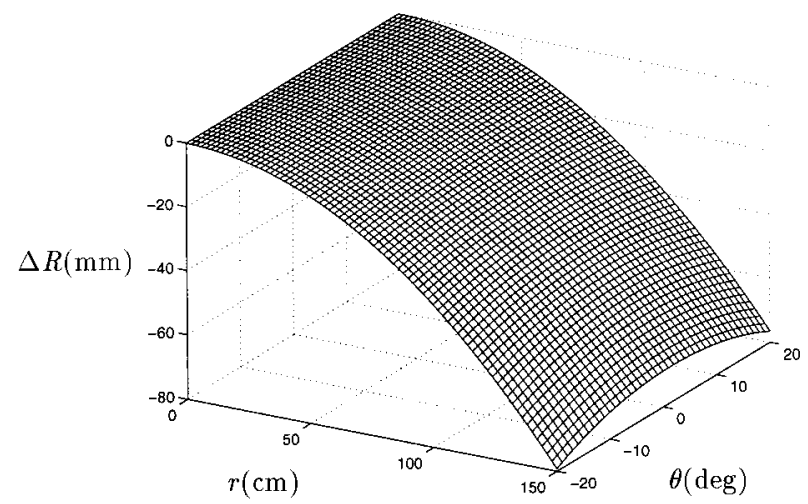

(c)

FIG. 8. Sensitivity of $R$ to (a) $d$, (b) $R$ and (c) $\theta$ when $\Delta z_{r}=0.18 \mathrm{~mm}$.

\section{SIMULATION RESULTS}

In the simulations, the signals are modeled according to Eq. (4) using the parameter values $A_{\max }=1, r_{\min }=5.8 \mathrm{~cm}$, $\rho_{c}=0.45 R-0.022, f_{0}=49.4 \mathrm{kHz}, c=343.3 \mathrm{~m} / \mathrm{s}$. Zero-mean white Gaussian noise $N(0, \sigma)$ of varying magnitude has been added to the signals before estimating the time-of-flight.

The initial estimate of the radius of curvature is made using Eq. (10) with the first set of measurements obtained at the initial (flat) position of the sensor (Fig. 2). The target position $(r, \theta, \phi)$ is estimated using Eqs. (11)-(13). Using these initial estimates, transducers will be rotated toward the object so that it is located along their line-of-sights in order to obtain more nearly accurate results. For this purpose, the inclination angles $\theta_{r}, \theta_{l}$ of the right and left transducers with respect to the target are estimated from the initial noisy measurements: 


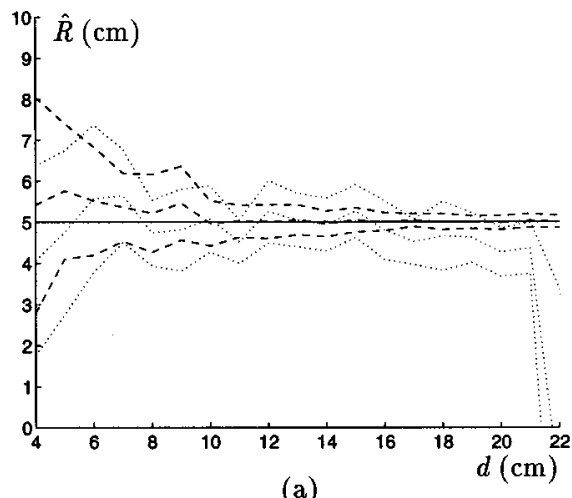

(a)

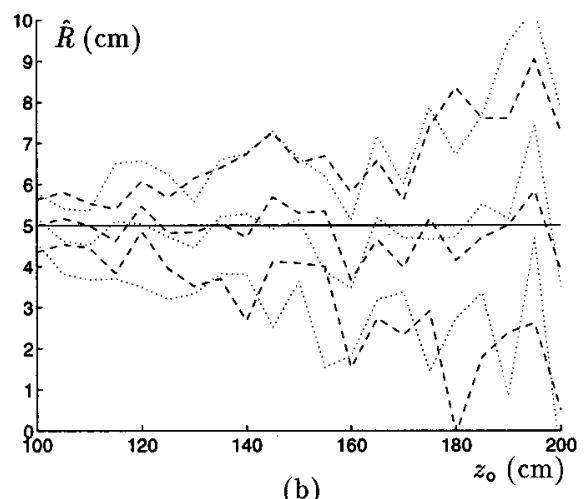

(b)

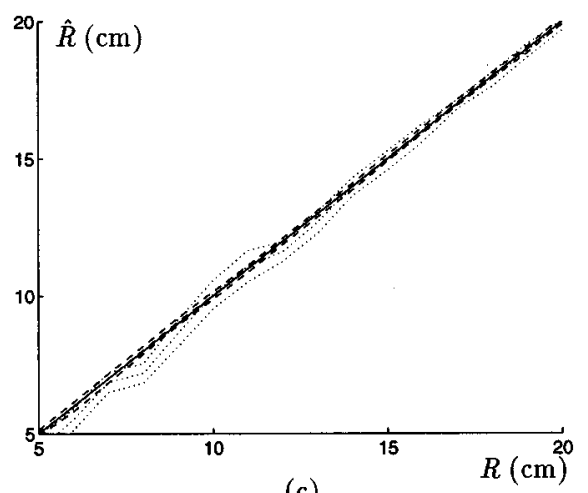

(c)

FIG. 9. Estimated radius versus (a) $d$, (b) $z_{0}$, and (c) $R$. Dotted and dashed lines indicate the mean of the estimate and $\pm \sigma_{\hat{R}}$ obtained at the flat and adapted positions, respectively. In part (a), $R=5 \mathrm{~cm}, z_{0}=100 \mathrm{~cm}$. In part (b), $R=5 \mathrm{~cm}, d=10 \mathrm{~cm}$. In part (c), $d=20 \mathrm{~cm}, z_{0}=100 \mathrm{~cm}$.

$$
\begin{aligned}
& \hat{\theta}_{r}=\tan ^{-1}\left(\frac{\hat{r} \cos \hat{\phi} \sin \hat{\theta}}{\hat{r} \cos \hat{\phi} \sin \hat{\theta}-d}\right), \\
& \hat{\theta}_{l}=\tan ^{-1}\left(\frac{\hat{r} \cos \hat{\phi} \sin \hat{\theta}}{\hat{r} \cos \hat{\phi} \sin \hat{\theta}+d}\right) .
\end{aligned}
$$

First, the central, right and left transducers are all rotated by $\hat{\phi}$ in elevation, and then rotated by $\hat{\theta}, \hat{\theta}_{r}$, and $\hat{\theta}_{l}$, respectively, so that they are approximately perpendicular to the object surface. New measurements are made and the radius of curvature is estimated for the second time, again using Eq. (10). Flat and rotated configurations of the transducers are illustrated in Fig. 2 in 2-D. The distance measurements $z_{0}, z_{r}, z_{l}, z_{u}, z_{d}$ should ideally be the same before and af-
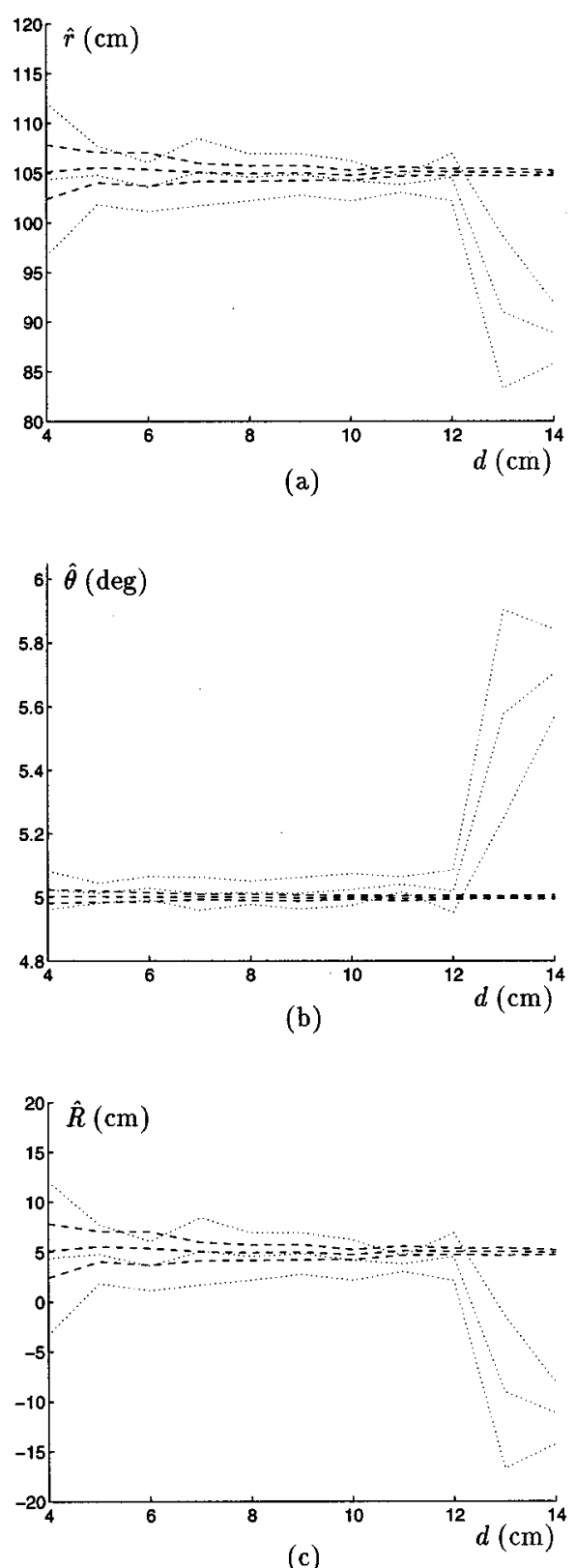

FIG. 10. Location and radius of curvature estimation versus $d$. Dotted and dashed lines indicate the mean of the estimate and $\pm \sigma_{\hat{R}}$ obtained at the flat and adapted positions respectively. In all parts of the figure, $R=5 \mathrm{~cm}, z_{0}$ $=100 \mathrm{~cm}$, and $\theta=5^{\circ}$.

ter the rotation. In practice, they will be slightly different due to the additive measurement noise. In the second estimate, the distance measurements $z_{0}, z_{r}, z_{l}, z_{u}, z_{d}$ are obtained with a larger SNR since the transducers are now looking straight ahead at the object. This in turn results in more nearly accurate estimates.

In all of the simulations, a 100-realization Monte-Carlo study has been employed. Figure 9(a) illustrates the effect of varying $d$ on the curvature estimate before and after the transducers are rotated. Note the reduction in the standard deviations of the estimates as $d$ is increased. For $d>21 \mathrm{~cm}$, all of the transducers cannot detect the object and the estimates become very erroneous. Figure 9(b) illustrates that as $z_{0}$ increases, standard deviations of both estimates increase. 


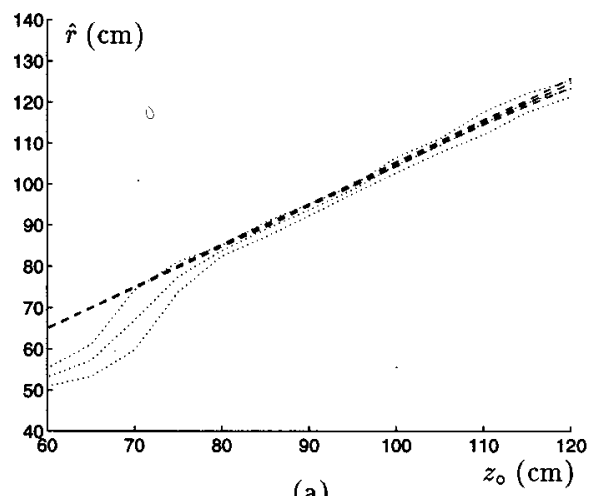

(a)

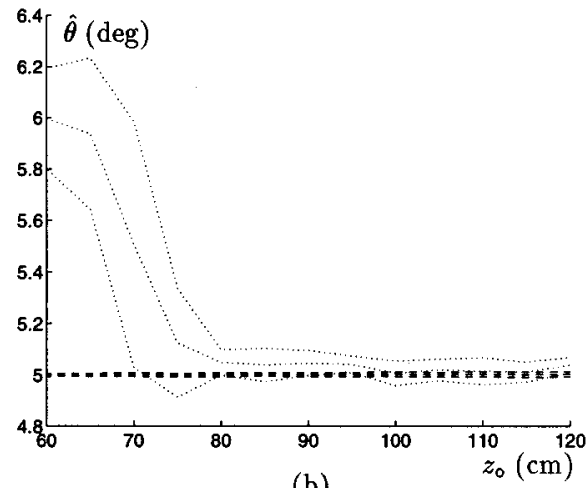

(b)

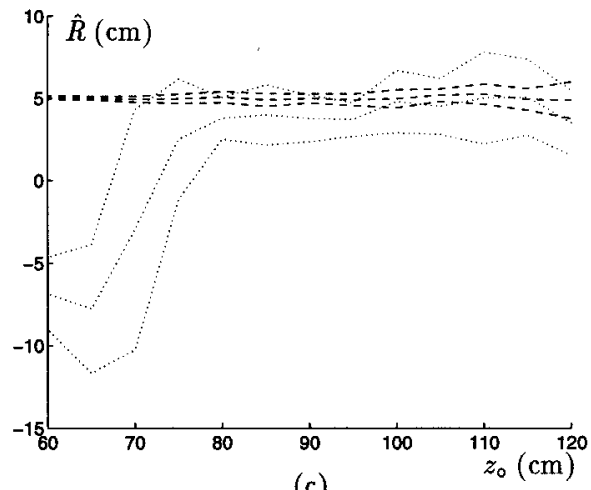

(c)

FIG. 11. Location and radius of curvature estimation versus $z_{0}$. Dotted and dashed lines indicate the mean of the estimate and $\pm \sigma_{\hat{R}}$ obtained at the flat and adapted positions, respectively. In all parts of the figure, $R=5 \mathrm{~cm}, d$ $=10 \mathrm{~cm}, z_{0}=100 \mathrm{~cm}$, and $\theta=5^{\circ}$.

Figure 9(c) illustrates the effect of the true radius. In all cases, significant improvement can be observed after the transducers are adapted.

Figure 10 shows the dependence of the estimates on $d$ for $R=5 \mathrm{~cm}, z_{0}=100 \mathrm{~cm}$, and $\theta=5^{\circ}$. Figure 10 (a) illustrates that as $d$ increases, the estimate after rotation keeps improving. The estimation before rotation improves up to $d$ $=12 \mathrm{~cm}$, and after that point, it gets worse since the target is now located either at very low-SNR regions of the sensitivity region or outside it (as $d$ increases, the normal angles of the left and right sensors increase). The average error in $\hat{R}$ before rotation is $5.96 \%$. After rotation, the error is reduced to $0.05 \%$. Figure 10 (b) illustrates how the azimuth estimate $\hat{\theta}$ is affected by $d$. The average error figures before and after

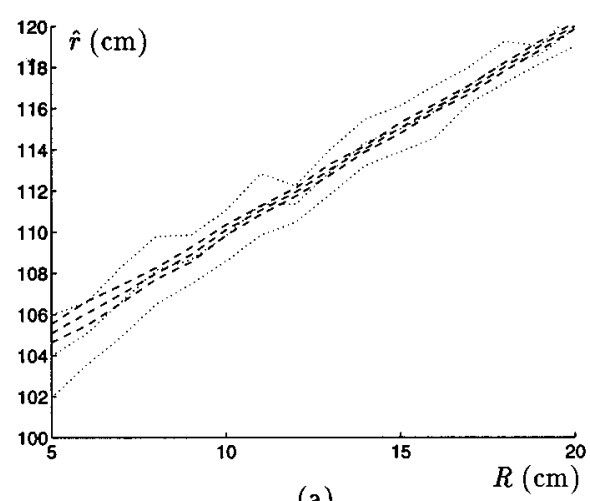

(a)

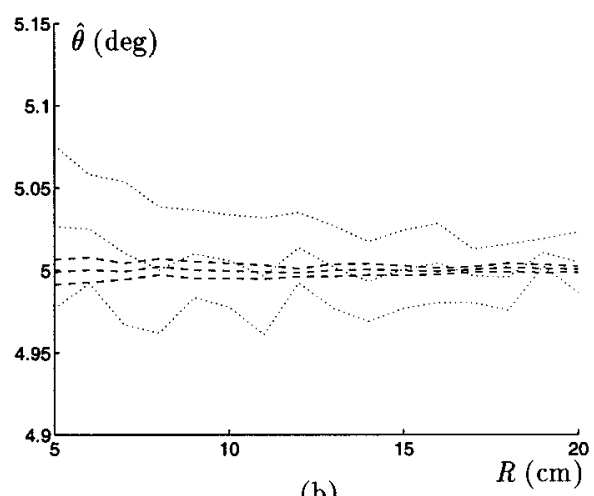

(b)

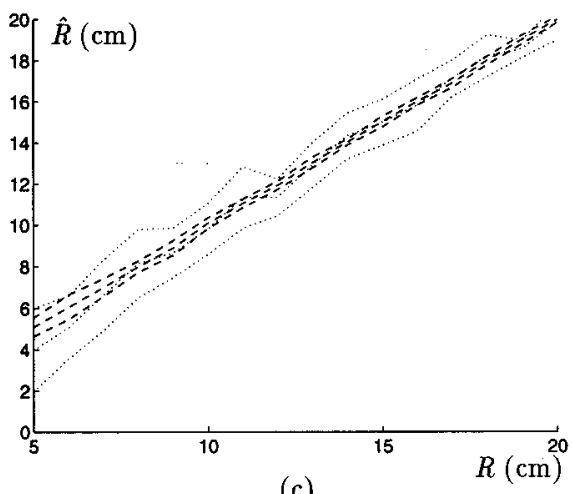

(c)

FIG. 12. Location and radius of curvature estimation versus $R$. Dotted and dashed lines indicate the mean of the estimate and $\pm \sigma_{\hat{R}}$ obtained at the flat and adapted positions, respectively. In all parts of the figure, $d=10 \mathrm{~cm}, z_{0}$ $=100 \mathrm{~cm}$, and $\theta=5^{\circ}$.

rotation are $6.0 \%$ and $0.0 \%$ respectively. Figure $10(\mathrm{c})$ illustrates the dependence of the curvature estimate on $d$. The average error before and after rotation is $11.4 \%$ and $1.2 \%$, respectively.

Figure 11 shows the dependence of $\hat{r}, \hat{\theta}$, and $\hat{R}$ estimates on the distance $z_{0}$ for $R=5 \mathrm{~cm}, d=10 \mathrm{~cm}, z_{0}=100 \mathrm{~cm}$, and $\theta=5^{\circ}$. For small values of $z_{0}$, the normal angles of the left and right sensors are large and the initial estimates obtained with the flat configuration are not very accurate. For large values of $z_{0}$, the normal angles decrease and the estimation accuracy improves. In Fig. 11(a), the average error of the distance estimate is $6.0 \%$ before rotation and $0.0 \%$ after rotation. In Fig. 11(c), the average error of radius of curvature estimation before rotation is $19.0 \%$ and $0.0 \%$ after rotation. 


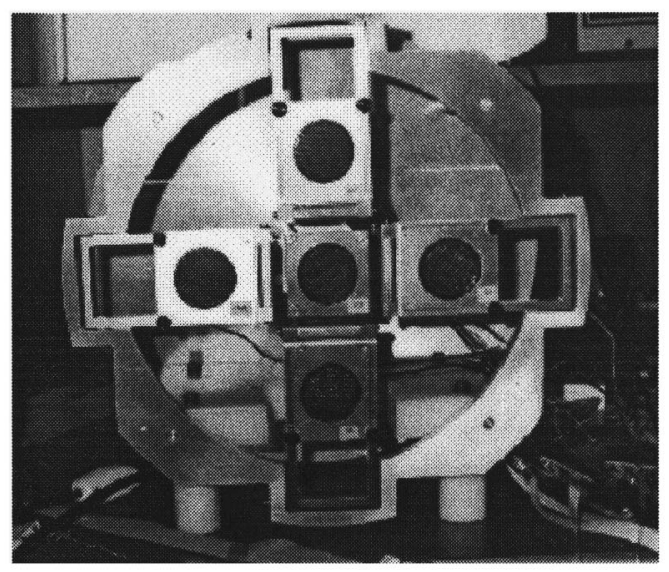

(a)

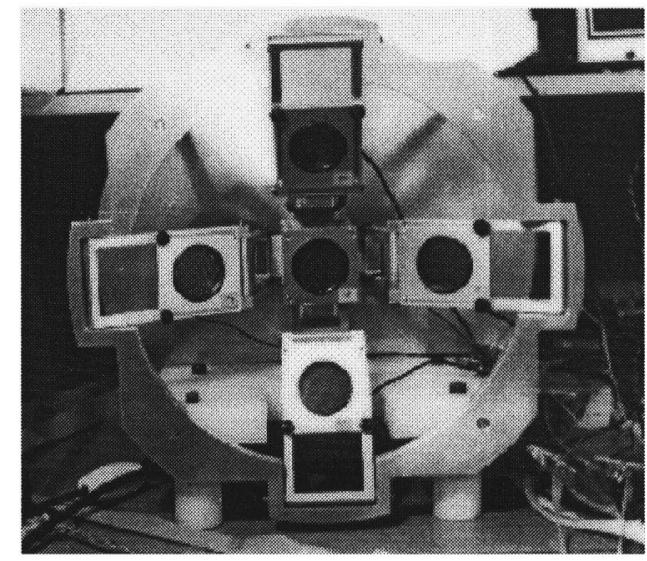

(b)

FIG. 13. Two extreme positions of the sensing device.

Again, there is a minimum value of $z_{0} \cong 70 \mathrm{~cm}$, at which the target is accurately detectable. For shorter distances, all estimates become erroneous since one or more transducers cannot detect the target.

Figure 12 shows the effect of varying $R$ on the $\hat{r}, \hat{\theta}$, and $\hat{R}$ estimates for $d=10 \mathrm{~cm}, z_{0}=100 \mathrm{~cm}$, and $\theta=5^{\circ}$. As $R$ increases, the accuracy of all three estimates improve. In addition, there is significant improvement after the transducers are adapted to the target, as evident from the reduction in the standard deviations of the estimates.

\section{EXPERIMENTAL VERIFICATION}

\section{A. The sensing device and the experimental setup}

The sensing device used in this investigation was precision constructed for 3-D sonar applications. A trade-off between simplicity of mechanical design and flexibility was established. The unit, illustrated in Fig. 13, consists of five Polaroid 6500 series acoustic transducers, each operating at a resonance frequency of $f_{0}=49.4 \mathrm{kHz} .{ }^{39}$ A central transducer is flanked by four transducers symmetrically. The position of the central transducer is fixed but the separation $d$ of each surrounding transducer from the center can be manually adjusted between 7.5 to $12.0 \mathrm{~cm}$.

The device has 16 mechanical joints which enable it to move with the aid of stepper motors located behind the central transducer. In one extreme position, all the sensors are coplanar [Fig. 13(a)]. In the other extreme, the flanking transducers have been rotated by $30^{\circ}$ as shown in Fig. 13(b). In between, there are 1020 intermediate positions. The flexibility of the sensor can be judiciously used to recognize 3-D targets and focus on them to use the sensor data more effectively.

The analysis of the previous sections has been verified by real sonar data from cylindrical and planar targets using a 4-channel DAS-50 A/D card with 12-bit resolution and $1 \mathrm{MHz}$ sampling frequency. Echo signals were processed on an IBM-PC 486 using the $\mathrm{C}$ programming language. The block diagram for the hardware is shown in Fig. 14. The experiments were conducted in 2-D to allow accurate cali- bration. Real distances were ascertained accurately by carrying out the whole set of experiments on large sheets of millimetric paper. Transducer separation was kept constant at $d=7.5 \mathrm{~cm}$ (except in the results presented in Table III where $d=12.0 \mathrm{~cm}$ and those in Table VII where $d$ is varied).

Each transducer was made to transmit and receive in sequence to avoid crosstalk and to benefit maximally from the high sampling rate of the A/D card. Starting at the transmit time, 10000 samples of each echo signal have been collected to estimate the time-of-flight. The targets employed in this study are: cylinders with radii $25 \mathrm{~mm}, 50 \mathrm{~mm}, 75 \mathrm{~mm}$ and a planar target. All targets used in the experiments were wooden, with smooth surfaces, each with a height of $120 \mathrm{~cm}$. In the experiments, the sensors were situated at the middle of this height so that the lower and upper edges of the cylinders remained outside the beam patterns of the transducers. For the maximum range considered in the experiments $(150 \mathrm{~cm})$, from the geometry, the beam extends over a transverse extent of $150 \times 2 \times \tan \alpha_{0}=64 \mathrm{~cm}$, which is less than $120 \mathrm{~cm}$. Since the edges remain outside the beam patterns, edge effects are not observed. The cylindrical target with radius $25 \mathrm{~mm}$ is considered a good approximation to an edge target since cylinders with small radii behave similarly to outer edges formed by the intersection of two planes. ${ }^{44}$

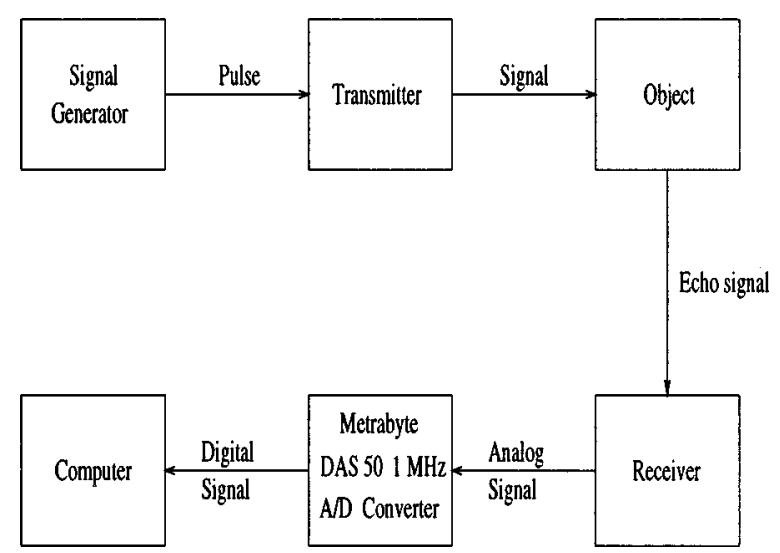

FIG. 14. The block diagram of the experimental setup. 
TABLE I. Experimental results for a cylinder of $R=75 \mathrm{~mm}$ when $d=7.5$ $\mathrm{cm}$.

\begin{tabular}{rrrrrrrrr}
\hline \hline $\begin{array}{c}z_{0} \\
(\mathrm{~mm})\end{array}$ & $\begin{array}{c}E\left\{\hat{z}_{0}\right\} \\
(\mathrm{mm})\end{array}$ & $\begin{array}{c}\sigma_{\hat{z}_{0}} \\
(\mathrm{~mm})\end{array}$ & $\begin{array}{r}E\{\hat{\theta}\} \\
(\mathrm{deg})\end{array}$ & $\begin{array}{c}\sigma_{\hat{\theta}} \\
(\mathrm{deg})\end{array}$ & $\begin{array}{r}E\left\{\hat{R}_{1}\right\} \\
(\mathrm{mm})\end{array}$ & $\begin{array}{r}\sigma_{\hat{R}_{1}} \\
(\mathrm{~mm})\end{array}$ & $\begin{array}{r}E\left\{\hat{R}_{2}\right\} \\
(\mathrm{mm})\end{array}$ & $\begin{array}{r}\sigma_{\hat{R}_{2}} \\
(\mathrm{~mm})\end{array}$ \\
\hline 300 & 299.93 & 0.14 & 0.01 & 0.08 & 73.39 & 7.78 & 74.47 & 4.67 \\
400 & 400.00 & 0.14 & 0.02 & 0.07 & 78.83 & 11.77 & 76.28 & 6.82 \\
500 & 500.15 & 0.14 & -0.04 & 0.07 & 75.14 & 17.13 & 75.16 & 9.76 \\
600 & 600.02 & 0.14 & -0.04 & 0.07 & 75.65 & 24.39 & 75.99 & 14.14 \\
700 & 700.10 & 0.18 & 0.01 & 0.07 & 81.72 & 32.00 & 78.87 & 18.24 \\
800 & 800.15 & 0.14 & 0.02 & 0.07 & 80.30 & 38.76 & 77.14 & 22.10 \\
900 & 900.42 & 0.14 & 0.07 & 0.07 & 71.12 & 50.33 & 73.62 & 28.18 \\
1000 & 1000.24 & 0.16 & -0.02 & 0.07 & 76.25 & 60.24 & 75.39 & 34.33 \\
1100 & 1100.23 & 0.14 & -0.03 & 0.07 & 75.55 & 70.94 & 76.24 & 39.02 \\
1200 & 1200.07 & 0.14 & 0.02 & 0.07 & 80.50 & 84.47 & 79.41 & 46.46 \\
1300 & 1300.19 & 0.14 & 0.00 & 0.07 & 78.71 & 95.51 & 76.33 & 53.49 \\
1400 & 1400.37 & 0.14 & 0.01 & 0.07 & 80.08 & 124.35 & 78.85 & 68.40 \\
1500 & 1500.22 & 0.15 & 0.01 & 0.07 & 69.19 & 118.16 & 75.39 & 66.17 \\
\hline \hline
\end{tabular}

\section{B. Experimental results}

Each target's surface distance $z_{0}$ to the central transducer was varied between 30 and $150 \mathrm{~cm}$ at $10 \mathrm{~cm}$ intervals. At each distance, data were collected while the target was stationary at $\theta=0^{\circ}$. For the same target position, 1000 sets of measurements were taken. Each set of measurements provides a single estimate of target radius of curvature, range and azimuth. The typical differential distance measured by the central and the right/left transducers varies between 0 and $10 \mathrm{~mm}$ depending on the target curvature and distance, for $d=7.5 \mathrm{~cm}$. As the range of the target increases, the differential becomes less reliable to extract the curvature information.

The means and standard deviations of $\hat{z}_{0}, \hat{\theta}$, and $\hat{R}$ of each type of target considered are computed and tabulated in Tables I-V. In all of the tables, results for $\hat{z}_{0}$ and $\hat{R}$ are tabulated individually instead of $r$ itself, which is the sum of these two components. Radius of curvature estimates have been presented both at the flat position and adapted position of the transducers. The results before and after adaptation are denoted by the subscripts 1 and 2, respectively. The results for $\hat{z}_{0}$ and $\hat{\theta}$ do not vary much after the rotation. Therefore,

TABLE II. Experimental results for a cylinder of $R=50 \mathrm{~mm}$ when $d=7.5$ $\mathrm{cm}$.

\begin{tabular}{rrrrrrrrr}
\hline \hline $\begin{array}{c}z_{0} \\
(\mathrm{~mm})\end{array}$ & $\begin{array}{c}E\left\{\hat{z}_{0}\right\} \\
(\mathrm{mm})\end{array}$ & $\begin{array}{c}\sigma_{\hat{z}_{0}} \\
(\mathrm{~mm})\end{array}$ & $\begin{array}{r}E\{\hat{\theta}\} \\
(\mathrm{deg})\end{array}$ & $\begin{array}{c}\sigma_{\hat{\theta}} \\
(\mathrm{deg})\end{array}$ & $\begin{array}{r}E\left\{\hat{R}_{1}\right\} \\
(\mathrm{mm})\end{array}$ & $\begin{array}{r}\sigma_{\hat{R}_{1}} \\
(\mathrm{~mm})\end{array}$ & $\begin{array}{r}E\left\{\hat{R}_{2}\right\} \\
(\mathrm{mm})\end{array}$ & $\begin{array}{r}\sigma_{\hat{R}_{2}} \\
(\mathrm{~mm})\end{array}$ \\
\hline 300 & 300.00 & 0.14 & -0.03 & 0.09 & 48.27 & 6.84 & 48.93 & 4.10 \\
400 & 399.79 & 0.14 & -0.02 & 0.07 & 51.13 & 10.08 & 50.27 & 6.25 \\
500 & 500.05 & 0.16 & -0.04 & 0.07 & 49.17 & 15.23 & 49.44 & 9.29 \\
600 & 600.59 & 0.17 & 0.04 & 0.07 & 52.33 & 22.43 & 51.79 & 13.24 \\
700 & 699.98 & 0.15 & 0.01 & 0.07 & 44.96 & 27.61 & 46.63 & 16.57 \\
800 & 799.87 & 0.14 & -0.03 & 0.07 & 44.55 & 35.02 & 48.36 & 21.52 \\
900 & 900.40 & 0.15 & 0.05 & 0.07 & 50.64 & 45.42 & 50.25 & 26.80 \\
1000 & 1000.39 & 0.14 & 0.03 & 0.07 & 59.87 & 57.41 & 55.48 & 33.39 \\
1100 & 1100.17 & 0.15 & -0.10 & 0.07 & 53.83 & 64.65 & 52.90 & 38.14 \\
1200 & 1200.14 & 0.14 & 0.01 & 0.07 & 48.08 & 83.71 & 49.21 & 47.71 \\
1300 & 1300.15 & 0.14 & -0.05 & 0.07 & 63.70 & 91.43 & 58.88 & 52.12 \\
1400 & 1400.63 & 0.15 & -0.08 & 0.07 & 40.92 & 122.53 & 45.62 & 69.84 \\
1500 & 1500.18 & 0.15 & 0.01 & 0.07 & 46.55 & 125.46 & 49.31 & 74.02 \\
\hline \hline
\end{tabular}

TABLE III. Experimental results for a cylinder of $R=50 \mathrm{~mm}$ with $d$ $=12.0 \mathrm{~cm}$.

\begin{tabular}{rcccccccc}
\hline \hline $\begin{array}{c}z_{0} \\
(\mathrm{~mm})\end{array}$ & $\begin{array}{r}E\left\{\hat{z}_{0}\right\} \\
(\mathrm{mm})\end{array}$ & $\begin{array}{c}\sigma_{\hat{z}_{0}} \\
(\mathrm{~mm})\end{array}$ & $\begin{array}{r}E\{\hat{\theta}\} \\
(\mathrm{deg})\end{array}$ & $\begin{array}{c}\sigma_{\hat{\theta}} \\
(\mathrm{deg})\end{array}$ & $\begin{array}{r}E\left\{\hat{R}_{1}\right\} \\
(\mathrm{mm})\end{array}$ & $\begin{array}{r}\sigma_{\hat{R}_{1}} \\
(\mathrm{~mm})\end{array}$ & $\begin{array}{r}E\left\{\hat{R}_{2}\right\} \\
(\mathrm{mm})\end{array}$ & $\begin{array}{c}\sigma_{\hat{R}_{2}} \\
(\mathrm{~mm})\end{array}$ \\
\hline 300 & $\ldots$ & $\ldots$ & $\cdots$ & $\cdots$ & $\ldots$ & $\ldots$ & $\ldots$ & $\cdots$ \\
400 & $\ldots$ & $\ldots$ & $\ldots$ & $\ldots$ & $\ldots$ & $\ldots$ & $\ldots$ & $\ldots$ \\
500 & 499.75 & 0.14 & -0.07 & 0.05 & 48.24 & 6.21 & 48.50 & 3.75 \\
600 & 599.97 & 0.17 & -0.08 & 0.05 & 49.54 & 8.96 & 49.08 & 5.47 \\
700 & 700.10 & 0.15 & 0.00 & 0.05 & 47.20 & 11.43 & 48.16 & 7.20 \\
800 & 799.93 & 0.15 & 0.02 & 0.05 & 48.58 & 15.21 & 48.01 & 9.13 \\
900 & 900.20 & 0.16 & 0.00 & 0.05 & 52.20 & 19.08 & 50.33 & 11.07 \\
1000 & 999.77 & 0.15 & -0.02 & 0.05 & 46.84 & 22.51 & 48.84 & 13.28 \\
1100 & 1100.43 & 0.16 & -0.03 & 0.05 & 55.43 & 27.45 & 53.69 & 15.37 \\
1200 & 1200.11 & 0.16 & -0.03 & 0.05 & 45.14 & 31.74 & 47.83 & 18.41 \\
1300 & 1300.34 & 0.15 & -0.02 & 0.04 & 48.48 & 38.63 & 48.50 & 21.63 \\
1400 & 1400.26 & 0.17 & -0.02 & 0.05 & 47.48 & 43.53 & 49.06 & 25.81 \\
1500 & 1500.37 & 0.14 & -0.07 & 0.04 & 49.37 & 46.80 & 50.29 & 25.74 \\
\hline \hline
\end{tabular}

for these parameters, only the initial estimates made at the flat position have been presented.

In Table III, results for $z_{0}=300 \mathrm{~mm}$ and $z_{0}=400 \mathrm{~mm}$ have not been presented since a very thin cylinder at $\theta=0^{\circ}$ is not detectable by the right and left transducers at the transducer separation of $d=12.0 \mathrm{~cm}$. For the same reason, results for $z_{0}=300 \mathrm{~mm}$ in Table IV are excluded when $d$ was set equal to $7.5 \mathrm{~cm}$. From the results, it can be observed that the $\hat{z}_{0}$ and $\hat{\theta}$ estimates are quite accurate: For a stationary target, the typical standard deviation of $\hat{z}_{0}$ is $0.18 \mathrm{~mm}$, which is approximately the resolution allowed by the A/D converter sampling rate. The typical standard deviation of the azimuth estimate is $0.08^{\circ}$. Error on $\hat{z}_{0}$ and $\hat{\theta}$ are relatively constant as the distance of the target is varied between 30 and $150 \mathrm{~cm}$. However, for the curvature, typical error is around 7-9 $\mathrm{mm}$ at $30 \mathrm{~cm}$, but keeps increasing with range for a fixed transducer separation. This is due to the reduction in the $d / r$ ratio which provides poorer resolution in estimating curvature. To estimate the curvature of a cylindrical target reliably, it is necessary to increase the transducer separation as the range is increased as seen in Fig. 6(a). To illustrate the effect of transducer separation, results for the maximum allowed separation in the system $(d=12 \mathrm{~cm})$ are included in

TABLE IV. Experimental results for a cylinder of $R=25 \mathrm{~mm}$ when $d$ $=7.5 \mathrm{~cm}$.

\begin{tabular}{rcccccccc}
\hline \hline $\begin{array}{c}z_{0} \\
(\mathrm{~mm})\end{array}$ & $\begin{array}{c}E\left\{\hat{z}_{0}\right\} \\
(\mathrm{mm})\end{array}$ & $\begin{array}{c}\sigma_{\hat{z}_{0}} \\
(\mathrm{~mm})\end{array}$ & $\begin{array}{r}E\{\hat{\theta}\} \\
(\mathrm{deg})\end{array}$ & $\begin{array}{c}\sigma_{\hat{\theta}} \\
(\mathrm{deg})\end{array}$ & $\begin{array}{c}E\left\{\hat{R}_{1}\right\} \\
(\mathrm{mm})\end{array}$ & $\begin{array}{c}\sigma_{\hat{R}_{1}} \\
(\mathrm{~mm})\end{array}$ & $\begin{array}{c}E\left\{\hat{R}_{2}\right\} \\
(\mathrm{mm})\end{array}$ & $\begin{array}{c}\sigma_{\hat{R}_{2}} \\
(\mathrm{~mm})\end{array}$ \\
\hline 300 & $\ldots$ & $\ldots$ & $\ldots$ & $\ldots$ & $\ldots$ & $\ldots$ & $\ldots$ & $\ldots$ \\
400 & 400.06 & 0.16 & 0.02 & 0.08 & 21.07 & 9.14 & 23.83 & 5.02 \\
500 & 500.11 & 0.14 & -0.03 & 0.07 & 23.58 & 14.00 & 22.49 & 7.84 \\
600 & 599.99 & 0.15 & 0.04 & 0.07 & 28.58 & 20.07 & 27.25 & 10.82 \\
700 & 699.99 & 0.15 & -0.05 & 0.07 & 26.20 & 26.01 & 24.67 & 13.53 \\
800 & 799.77 & 0.16 & 0.02 & 0.07 & 26.51 & 34.23 & 25.50 & 19.17 \\
900 & 899.91 & 0.20 & 0.02 & 0.07 & 30.37 & 42.98 & 27.94 & 22.78 \\
1000 & 999.44 & 0.24 & 0.03 & 0.08 & 29.86 & 57.48 & 26.90 & 34.49 \\
1100 & 1100.18 & 0.17 & 0.02 & 0.07 & 28.52 & 64.30 & 24.32 & 35.37 \\
1200 & 1200.44 & 0.16 & -0.04 & 0.07 & 23.47 & 78.03 & 27.65 & 41.37 \\
1300 & 1300.31 & 0.18 & -0.04 & 0.07 & 23.67 & 91.49 & 23.80 & 51.95 \\
1400 & 1400.01 & 0.15 & 0.00 & 0.07 & 23.50 & 98.58 & 24.37 & 55.01 \\
1500 & 1499.39 & 0.25 & 0.03 & 0.08 & 23.01 & 139.20 & 26.22 & 76.26 \\
\hline \hline
\end{tabular}


TABLE V. Experimental results for a planar target of $R=\infty$ when $d=7.5$ $\mathrm{cm}$.

\begin{tabular}{rrrrrrr}
\hline \hline $\begin{array}{c}z_{0} \\
(\mathrm{~mm})\end{array}$ & $\begin{array}{c}E\left\{\hat{z}_{0}\right\} \\
(\mathrm{mm})\end{array}$ & $\begin{array}{c}\sigma_{\hat{z}_{0}} \\
(\mathrm{~mm})\end{array}$ & $\begin{array}{r}E\{\hat{\theta}\} \\
(\mathrm{deg})\end{array}$ & $\begin{array}{c}\sigma_{\hat{\theta}} \\
(\mathrm{deg})\end{array}$ & $\begin{array}{c}E\left\{\hat{R}_{1}\right\}=E\left\{\hat{R}_{2}\right\} \\
(\mathrm{mm})\end{array}$ & $\begin{array}{c}\sigma_{\hat{R}_{1}}=\sigma_{\hat{R}_{2}} \\
(\mathrm{~mm})\end{array}$ \\
\hline 300 & 299.88 & 0.14 & -0.02 & 0.08 & $4.70 \times 10^{18}$ & $2.12 \times 10^{19}$ \\
400 & 399.94 & 0.14 & 0.01 & 0.08 & $1.30 \times 10^{19}$ & $3.36 \times 10^{19}$ \\
500 & 500.09 & 0.15 & -0.04 & 0.08 & $8.00 \times 10^{18}$ & $2.72 \times 10^{19}$ \\
600 & 600.11 & 0.16 & -0.01 & 0.07 & $1.42 \times 10^{19}$ & $3.49 \times 10^{19}$ \\
700 & 699.98 & 0.16 & -0.02 & 0.08 & $1.53 \times 10^{19}$ & $3.60 \times 10^{19}$ \\
800 & 800.24 & 0.15 & -0.06 & 0.08 & $8.00 \times 10^{18}$ & $2.71 \times 10^{19}$ \\
900 & 899.83 & 0.17 & 0.03 & 0.08 & $1.08 \times 10^{19}$ & $3.10 \times 10^{19}$ \\
1000 & 1000.07 & 0.15 & 0.03 & 0.08 & $1.25 \times 10^{19}$ & $3.31 \times 10^{19}$ \\
1100 & 1100.18 & 0.16 & 0.04 & 0.08 & $1.23 \times 10^{19}$ & $3.28 \times 10^{19}$ \\
1200 & 1199.92 & 0.17 & -0.04 & 0.08 & $7.90 \times 10^{18}$ & $2.70 \times 10^{19}$ \\
1300 & 1300.00 & 0.18 & -0.05 & 0.08 & $9.20 \times 10^{18}$ & $2.89 \times 10^{19}$ \\
1400 & 1399.80 & 0.15 & -0.02 & 0.08 & $1.36 \times 10^{19}$ & $3.43 \times 10^{19}$ \\
1500 & 1500.34 & 0.20 & 0.00 & 0.08 & $1.07 \times 10^{19}$ & $3.09 \times 10^{19}$ \\
\hline \hline
\end{tabular}

Table III. Compared to Table II where $d=7.5 \mathrm{~cm}$, it is observed that errors in the radius of curvature estimate are approximately reduced by $60 \%$. In Table $\mathrm{V}$, results for a planar target $(R=\infty)$ are illustrated. In the experiments, whenever the denominator of Eq. (10) is zero, a very large value $\left(10^{20}\right)$ is assigned to $R$ to be able to represent it numerically.

In Table VI, results for the cylinder with $R=25 \mathrm{~mm}$ are provided for $\theta=0^{\circ}, 3^{\circ}, 5^{\circ}, 8^{\circ}$. It is observed that the accuracies of range and azimuth estimates do not change significantly as compared to the case when the target is along the line-of-sight. The accuracy of the initial curvature estimate degrades with $|\theta|$ as expected. However, the estimates with the adapted configuration for target at different $\theta$ are comparable in accuracy. For larger values of $\theta$ than considered in the table, it is not possible to estimate the curvature since the target will be outside the sensitivity region of either the right or the left transducer.

Finally, the transducers were detached from the mounting and were placed on polyamid stands so that larger transducer separations than allowed by the prototype system could be tested $(d: 15.0-30.0 \mathrm{~cm})$. The results are presented in Table VII. When $d>21 \mathrm{~cm}$, it is not possible to acquire data with the side transducers at the flat position since the target at $z_{0}=1.00 \mathrm{~m}$ remains outside the joint sensitivity region of the transducers. Therefore, for these cases, the transducers are maintained approximately perpendicular to the object surface while experimental data are being collected.

Overall, the results indicate that the accuracy of the curvature estimation after adapting the transducers brings an improvement varying between $35 \%$ and $45 \%$.

TABLE VI. Experimental results for a cylinder with $R=25 \mathrm{~mm}$ for varying $\theta$ when $d=7.5 \mathrm{~cm}$.

\begin{tabular}{crccccccc}
\hline \hline $\begin{array}{c}\theta \\
(\mathrm{deg})\end{array}$ & $\begin{array}{c}E\left\{\hat{z}_{0}\right\} \\
(\mathrm{mm})\end{array}$ & $\begin{array}{c}\sigma_{\hat{z}_{0}} \\
(\mathrm{~mm})\end{array}$ & $\begin{array}{c}E\{\hat{\theta}\} \\
(\mathrm{deg})\end{array}$ & $\begin{array}{c}\sigma_{\hat{\theta}} \\
(\mathrm{deg})\end{array}$ & $\begin{array}{c}E\left\{\hat{R}_{1}\right\} \\
(\mathrm{mm})\end{array}$ & $\begin{array}{c}\sigma_{\hat{R}_{1}} \\
(\mathrm{~mm})\end{array}$ & $\begin{array}{c}E\left\{\hat{R}_{2}\right\} \\
(\mathrm{mm})\end{array}$ & $\begin{array}{c}\sigma_{\hat{R}_{2}} \\
(\mathrm{~mm})\end{array}$ \\
\hline 0 & 1000.26 & 0.16 & -0.04 & 0.07 & 22.65 & 56.81 & 24.64 & 30.60 \\
3 & 999.46 & 0.15 & 2.73 & 0.08 & 24.57 & 58.50 & 26.77 & 31.92 \\
5 & 1000.67 & 0.15 & 4.98 & 0.07 & 25.64 & 61.16 & 23.43 & 29.75 \\
8 & 1000.05 & 0.16 & 7.53 & 0.07 & 27.34 & 63.34 & 25.06 & 31.42 \\
\hline \hline
\end{tabular}

TABLE VII. Experimental results for varying $d$ for a cylinder of radius $R$ $=75 \mathrm{~mm}$, located at $z_{0}=1.00 \mathrm{~m}$ and $\theta=0^{\circ}$.

\begin{tabular}{lrcrccccr}
\hline \hline $\begin{array}{c}d \\
(\mathrm{~cm})\end{array}$ & $\begin{array}{c}E\left\{\hat{z}_{0}\right\} \\
(\mathrm{mm})\end{array}$ & $\begin{array}{c}\sigma_{\hat{z}_{0}} \\
(\mathrm{~mm})\end{array}$ & $\begin{array}{r}E\left\{\hat{\theta}_{\}}\right\} \\
(\mathrm{deg})\end{array}$ & $\begin{array}{c}\sigma_{\hat{\theta}} \\
(\mathrm{deg})\end{array}$ & $\begin{array}{c}E\left\{\hat{R}_{1}\right\} \\
(\mathrm{mm})\end{array}$ & $\begin{array}{c}\sigma_{\hat{R}_{1}} \\
(\mathrm{~mm})\end{array}$ & $\begin{array}{c}E\left\{\hat{R}_{2}\right\} \\
(\mathrm{mm})\end{array}$ & $\begin{array}{c}\sigma_{\hat{R}_{2}} \\
(\mathrm{~mm})\end{array}$ \\
\hline 15 & 999.65 & 0.14 & 0.01 & 0.07 & 73.61 & 20.49 & 76.29 & 12.50 \\
20 & 1000.57 & 0.14 & -0.04 & 0.07 & 72.65 & 18.87 & 73.46 & 10.96 \\
25 & 999.48 & 0.16 & 0.02 & 0.08 & $\cdots$ & $\cdots$ & 77.61 & 9.75 \\
30 & 999.88 & 0.15 & 0.03 & 0.07 & $\cdots$ & $\cdots$ & 72.28 & 8.31 \\
35 & 999.26 & 0.13 & 0.05 & 0.07 & $\cdots$ & $\cdots$ & 75.73 & 6.24 \\
40 & 1000.74 & 0.15 & 0.04 & 0.06 & $\cdots$ & $\cdots$ & 74.05 & 5.49 \\
\hline \hline
\end{tabular}

\section{DISCUSSION AND CONCLUSION}

A sensing device capable of estimating the location and radius of curvature of spherical and cylindrical targets has been described. The main goal of the study is to assess the performance of radius of curvature estimation. The estimation accuracy can be improved by employing an adaptive sensor configuration: After acquiring the initial data, transducers are rotated to align their line-of-sights with the object. This way, SNR is increased and more nearly accurate estimates can be obtained. Two limiting cases are of special interest: the point (in 3-D) or line (in 2-D) target and the planar target. Analytical results are verified by real sonar data from cylindrical and planar targets. Typical accuracies in range and azimuth are $0.18 \mathrm{~mm}$ and $0.1^{\circ}$, respectively. Accuracy of the curvature estimate depends on the target type and system parameters such as transducer separation and operating range. The estimation with the adapted configuration gives much better results than without adaptation, and brings an improvement in the accuracy of about $40 \%$.

The radius of curvature estimation provides valuable information for differentiating reflectors with different radii (including $R=0$ for an edgelike reflector to $R=\infty$ for a planar reflector). The classification procedure, consistent with the experimental results, is illustrated in Fig. 15. The uncertainty region of each radius estimate is considered to be between $\left[\hat{R}-3 \sigma_{\hat{R}}, \hat{R}+3 \sigma_{\hat{R}}\right]$ assuming zero-mean Gaussiandistributed estimation error. The standard deviation $\sigma_{R}$ increases with the radius of curvature. Given two targets with constant curvature, if there is overlap between their uncertainty regions, then these targets may not be distinguished for estimates which fall within the overlap region, shown by

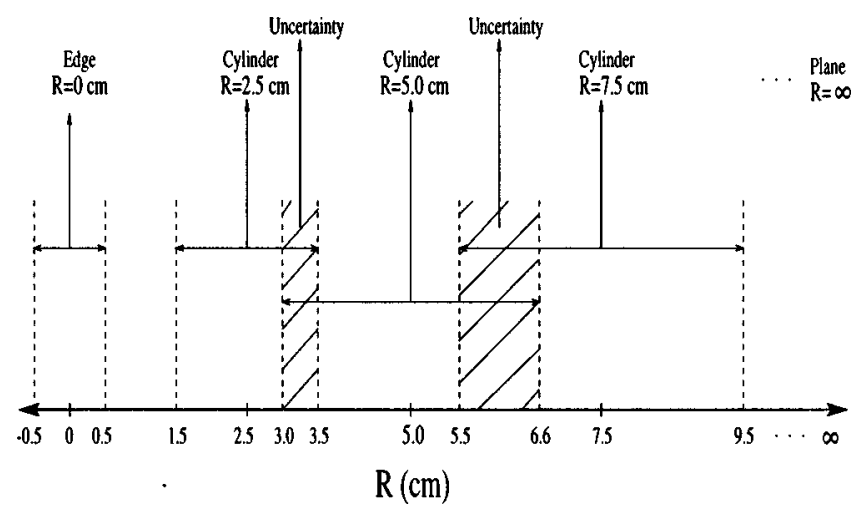

FIG. 15. Target discrimination using radius of curvature estimation. 
hatched areas in Fig. 15. The results presented allow such decisions to be based on a solid footing.

For reliable curvature estimation, it is necessary to increase the transducer separation as the range is increased. The transducer separation in the present system is relatively limited and not capable of real-time dynamic adaptation. A system which is adaptive also in this respect would be able to maintain high accuracy over a broader range of distances.

When dealing with shapes more general than cylinders and spheres, such as ellipsoidal surfaces, the geometry will be slightly more complicated. Nevertheless, a similar approach can be taken, possibly requiring additional sensors, or sensors with greater capability of motion. Finally, although the method has been developed for convex $(R>0)$ reflectors, it is equally applicable to concave $(R<0)$ reflectors. Targets that have spatially varying curvature which may become both concave and convex have also been addressed in recent work. ${ }^{45,46}$ Naturally, the larger the number of parameters (or degrees of freedom) of the surface, the larger the number of sensors needed.

\section{ACKNOWLEDGMENTS}

This work was supported by TÜBITAK under Project No. EEEAG-92 and the British Council Academic Link Program. The authors would like to thank the anonymous reviewers for their comments.

${ }^{1}$ G. C. Carter and E. R. Robinson, "'Ocean effects on time-delay estimation requiring adaptation,” IEEE J. Ocean Eng. 18, 367-378 (1993).

${ }^{2}$ A. C. Smith and G. C. L. Searle, "Empirical observations of a sonar adaptive array," IEE Proc. F, Commun. Radar Signal Process. 132, 595597 (1985).

${ }^{3}$ K. Scarbrough, G. C. Carter, and R. J. Tremblay, "Performance predictions for coherent and incoherent processing techniques of time-delay estimation,' IEEE Trans. Acoust., Speech, Signal Process. 31, 1191-1196 (1983).

${ }^{4}$ G. C. Carter, "Coherence and time-delay estimation," Proc. IEEE 75, 236-255 (1987).

${ }^{5} \mathrm{M}$. Wazenski and D. Alexandrou, "Active, wideband detection and localization in an uncertain multipath environment," J. Acoust. Soc. Am. 101, 1961-1970 (1997).

${ }^{6}$ D. J. W. Hardie and A. B. Gallaher, "Review of numerical methods for predicting sonar array performance," IEE Proc. F, Radar Sonar and Navigation 143, 196-203 (1996).

${ }^{7}$ R. Smith, A. Stevens, A. Frost, and P. Probert, "Developing a sensorbased underwater navigation system," Int. J. Syst. Sci. 29, 1145-1155 (1998).

${ }^{8}$ W. K. Stewart, "3-dimensional stochastic modeling using sonar sensing for undersea robotics," Autonomous Robots 3, 121-143 (1996).

${ }^{9}$ R. Kuc, "Three-dimensional tracking using qualitative bionic sonar,', Robotics Autonomous Syst. 11, 213-219 (1993).

${ }^{10}$ M. L. Hong and L. Kleeman, "Ultrasonic classification and location of 3-D room features using maximum likelihood estimation II,' Robotica 15, 645-652 (1997).

${ }^{11}$ L. Kleeman and H. Akbarally, "A sonar sensor for accurate 3-D target localization and classification," in Proceedings IEEE International Conference on Robotics and Automation, Nagoya, Japan, May 21-27 (IEEE, Piscataway, NJ, 1995), pp. 3003-3008.

${ }^{12}$ H. Peremans, K. Audenaert, and J. M. Van Campenhout, "A highresolution sensor based on tri-aural perception," IEEE Trans. Rob. Autom. 9, 36-48 (1993).

${ }^{13}$ A. M. Sabatini, "Statistical estimation algorithms for ultrasonic detection of surface features," in Proceedings IEEE/RSJ International Conference on Intelligent Robots and Systems, Munich, Germany, September 12-16 (IEEE, Piscataway, NJ, 1994), pp. 1845-1852.

${ }^{14}$ A. M. Sabatini, "Sampled baseband correlators for in-air ultrasonic rangefinders,"' IEEE Trans. Ind. Electron. 45, 341-350 (1998).
${ }^{15}$ M. K. Brown, "The extraction of curved surface features with generic range sensors,' Int. J. Robotics Res. 5, 3-18 (1986).

${ }^{16}$ R. Kuc, "Biologically motivated adaptive sonar system," J. Acoust. Soc. Am. 100, 1849-1854 (1996).

${ }^{17}$ R. Kuc, "Biomimetic sonar recognizes objects using binaural information," J. Acoust. Soc. Am. 102, 689-696 (1997).

${ }^{18}$ A. Hilton, J. Illingworth, and T. Windeatt, "Statistics of surface curvature estimates," Pattern Recogn. 28, 1201-1221 (1995).

${ }^{19}$ I. E. Dror, M. Zagaeski, and C. F. Moss, "3-dimensional target recognition via sonar-a neural network model," Neural Networks 8, 149-160 (1995).

${ }^{20}$ J. A. Simmons, P. A. Saillant, J. M. Wotton, T. Haresign, M. J. Ferragamo, and C. F. Moss, "Composition of biosonar images for target recognition by echolocating bats," Neural Networks 8, 1239-1261 (1995).

${ }^{21}$ H. L. Roitblat, W. W. L. Au, P. E. Nachtigall, R. Shizumura, and G. Moons, "Sonar recognition of targets embedded in sediment," Neural Networks 8, 1263-1273 (1995).

${ }^{22}$ R. P. Gorman and T. J. Sejnowski, "Learned classification of sonar targets using a massively parallel network," IEEE Trans. Acoust., Speech, Signal Process. 36, 1135-1140 (1998).

${ }^{23}$ T. Ogawa, K. Kameyama, R. Kuc, and Y. Kosugi, "Source localization with network inversion using an answer-in-weights scheme," IEICE Trans. Inf. Syst. E79-D, 608-619 (1996).

${ }^{24}$ W. W. L. Au, "Comparison of sonar discrimination-dolphin and artificial neural network," J. Acoust. Soc. Am. 95, 2728-2735 (1994).

${ }^{25}$ W. Chang, B. Bosworth, and G. C. Carter, "Results of using an artificial neural network to distinguish single echoes from multiple sonar echoes," J. Acoust. Soc. Am. 94, 1404-1408 (1993).

${ }^{26} \mathrm{~V}$. Tonard and J. Chatillon, "Acoustical imaging of extended targets by means of synthetic-aperture sonar technique," Acustica 83, 992-999 (1997).

${ }^{27}$ S. Stergiopoulos, "Implementation of adaptive and synthetic-aperture processing schemes in integrated active-passive sonar systems," Proc. IEEE 86, 358-396 (1998).

${ }^{28}$ J. Zemanek, "Beam behavior within the nearfield of a vibrating piston," J. Acoust. Soc. Am. 49, 181-191 (1971).

${ }^{29}$ A. D. Pierce, Acoustics, An Introduction to Its Physical Principles and Applications (McGraw-Hill, New York, 1981).

${ }^{30} \mathrm{P}$. M. Morse and K. U. Ingard, Theoretical Acoustics (McGraw-Hill, New York, 1968).

${ }^{31}$ L. W. Camp, Underwater Acoustics (Wiley-Interscience, New York, 1970), Chap. 7, p. 166.

${ }^{32} \mathrm{O}$. Bozma and R. Kuc, "Characterizing pulses reflected from rough surfaces using ultrasound," J. Acoust. Soc. Am. 89, 2519-2531 (1991).

${ }^{33}$ B. Ayrulu and B. Barshan, "Identification of target primitives with multiple decision-making sonars using evidential reasoning," Int. J. Robotics Res. 17, 598-623 (1998).

${ }^{34} \mathrm{~B}$. Ayrulu, "Classification of target primitives with sonar using two nonparametric data-fusion methods," Master's thesis, Bilkent University, Department of Electrical Engineering, Ankara, Turkey, July 1996.

${ }^{35}$ B. Barshan and R. Kuc, "A bat-like sonar system for obstacle localization,’' IEEE Trans. Syst. Man Cybern. 22, 636-646 (1992).

${ }^{36} \mathrm{~B}$. Barshan, A sonar-based mobile robot for batlike prey capture, Ph.D. thesis, Yale University, Department of Electrical Engineering, New Haven, CT, December 1991. University of Michigan Microfilms, order number 9224325 .

${ }^{37}$ W. H. Press, B. P. Flannery, S. A. Teukolsky, and W. T. Vetterling, Numerical Recipes in Pascal (Cambridge University Press, Cambridge, 1989), pp. 574-579.

${ }^{38} \mathrm{~B}$. Barshan and B. Ayrulu, "Performance comparison of four methods of time-of-flight estimation for sonar waveforms,' Electron. Lett. 34, 16161617 (1998).

${ }^{39}$ Polaroid Corporation, "Ultrasonic components group," 119 Windsor St., Cambridge, MA 02139 (1990).

${ }^{40} \mathrm{~B}$. Barshan and O. Arıkan, "Performance analysis of two linear array processing algorithms for point-obstacle localization," in Proceedings SPIE Signal and Data Processing of Small Targets, San Diego, CA, July 11-13, 1995, edited by O. E. Drummond (SPIE, Bellingham, WA, 1995), Vol. 2561, pp. 533-544.

${ }^{41}$ M. L. Hong and L. Kleeman, "Analysis of ultrasonic differentiation of three-dimensional corners, edges and planes," in Proceedings IEEE International Conference on Robotics and Automation, Nice, France, May 12- 
14, 1992 (IEEE Computer Society Press, Los Alamitos, CA 1992), pp. 580-584.

${ }^{42}$ J. J. Leonard and H. F. Durrant-Whyte, "Mobile robot localization by tracking geometric beacons," IEEE Trans. Rob. Autom. 7, 376-382 (1991).

${ }^{43}$ B. Barshan and R. Kuc, "Differentiating sonar reflections from corners and planes by employing an intelligent sensor,' IEEE Trans. Pattern. Anal. Mach. Intell. 12, 560-569 (1990).

${ }^{44}$ R. Kuc and M. W. Siegel, "Physically-based simulation model for acous- tic sensor robot navigation," IEEE Trans. Pattern. Anal. Mach. Intell. PAMI-9, 766-778 (1987).

${ }^{45}$ D. Başkent and B. Barshan, "Morphological surface profile extraction from multiple sonars,' in Proceedings of the 1998 IEEE/RSJ International Conference on Intelligent Robots and Systems, Victoria, B.C., Canada, October 1998 (IEEE, Piscataway, NJ, 1998), pp.1515-1520.

${ }^{46}$ D. Başkent and B. Barshan, "Surface profile determination from multiple sonar data using morphological processing,' Int. J. Robotics Res., in press. 